\title{
Single stellar populations in the near-infrared
}

\section{Preparation of the IRTF spectral stellar library ${ }^{\star}$}

\author{
S. Meneses-Goytia ${ }^{1}$, R. F. Peletier ${ }^{1}$, S. C. Trager ${ }^{1}$, J. Falcón-Barroso ${ }^{2,3}$, M. Koleva ${ }^{4}$, and A. Vazdekis ${ }^{2,3}$ \\ 1 Kapteyn Instituut, Rijksuniversiteit Groningen, Landleven 12, 9747AD Groningen, The Netherlands \\ e-mail: s.meneses-goytia@astro.rug.nl \\ 2 Instituto de Astrofísica de Canarias, via Láctea s/n, La Laguna, Tenerife, Spain \\ 3 Departamento de Astrofísica, Universidad de La Laguna, La Laguna, 38205 Tenerife, Spain \\ ${ }^{4}$ Sterrenkundig Observatorium, Ghent University, Krijgslaan 281, S9, 9000 Ghent, Belgium
}

Received 18 March 2014 / Accepted 20 June 2015

\begin{abstract}
We present a detailed study of the stars of the NASA InfraRed Telescope Facility (IRTF) spectral library to understand its full extent and reliability for use with stellar population (SP) modeling. The library consist of 210 stars, with a total of 292 spectra, covering the wavelength range of 0.94 to $2.41 \mu \mathrm{m}$ at a resolution $R \approx 2000$. For every star we infer the effective temperature $\left(T_{\text {eff }}\right)$, gravity (log $g$ ) and metallicity $\left(\left[Z / Z_{\odot}\right]\right)$ using a full-spectrum fitting approach in a section of the $K$-band (2.19 to $\left.2.34 \mu \mathrm{m}\right)$ and temperature-NIR colour relations. We test the flux calibration of these stars by calculating their integrated colours and comparing them with the Pickles library colour-temperature relations. We also investigate the NIR colours as a function of the calculated effective temperature and compared them in colour-colour diagrams with the Pickles library. This latter test shows a good broad-band flux calibration, important for the SP models. Finally, we measure the resolution $R$ as a function of wavelength. We find that the resolution increases as a function of lambda from about $6 \AA$ in $J$ to $10 \AA$ in the red part of the $K$-band. With these tests we establish that the IRTF library, the largest currently available general library of stars at intermediate resolution in the NIR, is an excellent candidate to be used in stellar population models. We present these models in the next paper of this series.
\end{abstract}

Key words. infrared: stars - stars: fundamental parameters - catalogs - methods: data analysis - techniques: spectroscopic stars: kinematics and dynamics

\section{Introduction}

The near-infrared (NIR) spectral region contains several features that provide information about the stellar content of galaxies. The spectra of stellar populations in the NIR are strongly influenced by cool, late-type stars. Red giant branch (RGB) stars are old ( $>2$ Gyr) stars that have a stronger contribution at older ages and also as a function of redder wavelengths. Thermally pulsating asymptotic giant branch (AGB) stars, on the other hand, contribute most to the integrated light of a stellar population between 1 and 3 Gyr. However, during the lifetime of a galaxy, we also find regular AGB stars contributing to the spectrum at all ages. Therefore, in order to create stellar population models for all galaxy ages, we need stellar libraries that include these stars.

Stellar libraries are compilations of stellar spectra with a certain wavelength range and resolution covering a large part of the parameter space of effective temperature, gravity and chemical abundances. These libraries can be either theoretical or empirical. Theoretical libraries are calculated using models and atmospheres that can be determined for a wide range of stellar parameters and detailed chemical abundances, at nearly unlimited

* The IRTF spectral library is available at irtfweb.ifa.hawaii. edu/spex/IRTF_Spectral_Library/ spectral resolution (e.g. Westera et al. 2002; Coelho et al. 2007; Allard et al. 2012, among others). Model stellar atmospheres, however, are at present not able to reproduce the full spectra of some observed stars as a result of systematic uncertainties in our understanding of stellar atmospheres and a lack of complete atomic and molecular line lists, especially at lower temperatures. Observational or empirical libraries are compilations of observations of real stars that come with instrumental limitations such as limited resolution, incomplete correction of atmospheric absorption, and since most of the spectra come from stars in the Galactic disk, they might not be fully adequate to study stellar populations in other environments, such as elliptical galaxies. Examples of often used stellar libraries include Lançon \& Wood (2000), Cenarro et al. (2001), and Sánchez-Blázquez et al. (2006).

Since hot stars dominate the light in the ultraviolet (UV), while cool stars are prominent in the NIR, it is advantageous to use a large wavelength range when studying the stellar populations of galaxies (Frogel et al. 1978; Maraston 2005). Until now, most empirical stellar libraries are found in the optical, while some are available in the near-UV, and only very few in the NIR. With these libraries it is possible to model stellar populations in globular clusters and galaxies in the optical in 
two ways, using line indices, mostly using the Lick-IDS system (e.g. Burstein et al. 1984; Worthey et al. 1994), and using full-spectrum fitting (FSF). Excellent full-spectrum fits have been made of dwarf galaxies (Koleva et al. 2009), elliptical galaxies (Yamada et al. 2006; Conroy \& van Dokkum 2012), and globular clusters (Schiavon et al. 2004). The fits of giant elliptical galaxies show that some strong lines, most prominently the $\mathrm{Mg} b$ line at $5177 \AA$, cannot be fit by stellar population models using empirical libraries, indicating that the $[\mathrm{Mg} / \mathrm{Fe}]$ ratios in these objects are different from that of the solar neighbourhood (Peterson 1977; Peletier 1989; Worthey et al. 1992). By now it is clear that many abundance ratios in galaxies are not the same as in the solar neighbourhood (e.g. Yamada et al. 2006; Conroy \& van Dokkum 2013). To first order galaxy spectra in the optical between 4000 and $6000 \AA$ are, however, fairly well understood.

Theoretical model atmospheres have become more and more detailed over the years, producing libraries in different wavelength ranges. The most complete libraries in the NIR include the BaSeL models (Lejeune et al. 1997, 1998; Westera et al. 2002), Tlusty models (Lanz \& Hubeny 2003, 2007), and the models by Aringer et al. (2009). Compendia of stellar-type spectra formed from spectra of stars of similar type have also been made, for example by Pickles (1998), that combine various observations providing standard spectra for all spectral types and luminosity classes. Empirical libraries in the NIR include Lançon \& Wood (2000), Cushing et al. (2005) and Rayner et al. (2009). Mouhcine \& Lançon (2002), Maraston et al. (2009) and Conroy et al. (2009) have made stellar population models based on these libraries.

Stellar spectra observed by Rayner et al. (2009) and Cushing et al. (2005), compiled in the IRTF spectral library allow the modelling of stellar populations in the NIR. Conroy et al. (2009) used a handful of spectra in this library and Meneses-Goytia \& Peletier (2012) have constructed preliminary SSP models with this library. The library is a powerful ingredient for stellar population models in the NIR owing to the large number of cool stars it contains. These stars contribute strongly in this wavelength range and their spectra are of higher resolution than their empirical predecessors in the same wavelength region such as those observed by Lançon \& Wood (2000). To be able to use the IRTF spectral library for stellar population synthesis, an accurate determination of the spectral calibration and associated stellar parameters of the stars in the library must be established. A well-calibrated stellar library, with an accurate set of stellar parameters and known resolution, leads to a reliable development of models. The information that can be extracted from these models, such as integrated colours are linked to the colours of the stars.

In this series of papers, we aim to understand more about the cool stellar populations in unresolved galaxies by building single stellar population (SSP) models in the NIR and comparing them to galaxy observations. In this paper, the first in this series, we characterise the spectra in the IRTF spectral library and determine the stellar parameters, $T_{\text {eff }}, \log g$ and metallicity $\left(\left[Z / Z_{\odot}\right]\right)$ of the stars, tied to the parameters in Cenarro et al. (2007), in Sect. 3. We test the flux calibration of the library and the behaviour of the integrated colours of its stars and their stellar parameters in Sect. 4. And finally, in Sect. 5, we measure the full width at half maximum (FWHM) and resolution of the library spectra. In Fig. 1, we show the wavelength ranges used for our analysis.

In our second paper (Meneses-Goytia et al. 2015, hereafter Paper II), we calculate SSP models following a similar approach

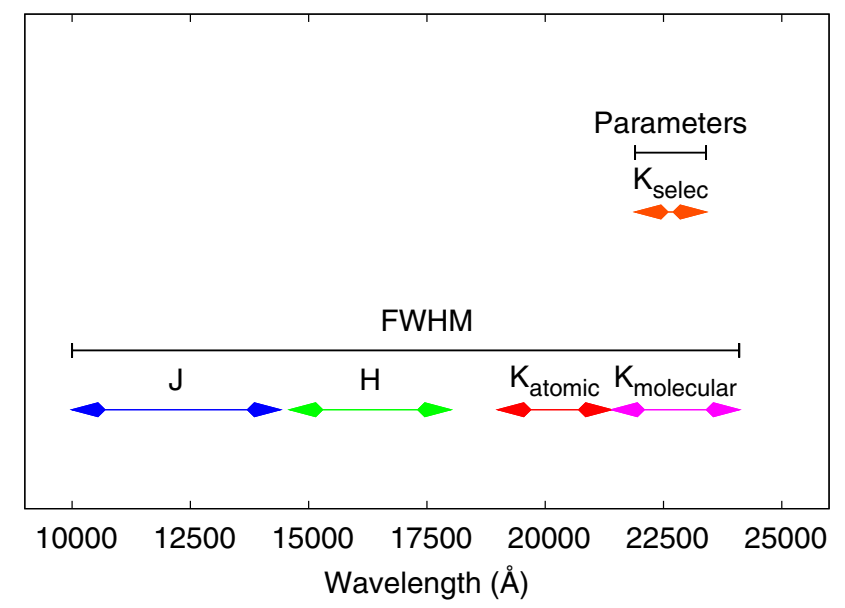

Fig. 1. Wavelength ranges used in this work. For the determination of the stellar parameters through FSF, we use a section of the $K$-band (2.19 to $2.34 \mu \mathrm{m}$, see Sect. 3.1). For the determination of the nominal spectral resolution (Sect. 5), we divide the wavelength range into four sections representing respectively the $J(1.04-1.44 \mu \mathrm{m})$ and $H(1.46-1.80 \mu \mathrm{m})$ bands, as well as the atomic $(1.90-2.14 \mu \mathrm{m})$ and molecular-dominated (2.14-2.41 $\mu \mathrm{m})$ ranges of the $K$-band.

to Vazdekis et al. (2010) but using the IRTF spectral library. We calculate full spectral energy distributions (SEDs) by finding a stellar spectrum with the appropriate stellar parameters $\left(T_{\text {eff }}\right.$, $\log g$ and metallicity) from the stellar library using interpolation for every point on a theoretical isochrone, weighting them by an initial mass function. In the third paper in the series Meneses-Goytia et al. (in prep., hereafter Paper III), we use our models to analyse a number of composite stellar systems.

\section{The IRTF spectral library}

The IRTF spectral library ${ }^{1}$ is a compilation of stellar spectra observed with the medium-resolution spectrograph SpeX at the NASA Infrared Telescope Facility on Mauna Kea (Rayner et al. 2009; Cushing et al. 2005). This library covers the NIR range from 0.8 to $2.5 \mu \mathrm{m}$ (and extends in some cases out to $5.2 \mu \mathrm{m}$ ). We focus on the spectral region for the $J, H$ and $K$-bands $(0.94$ to $2.41 \mu \mathrm{m})$. These spectra were observed at a resolving power of $R=2000(R=\lambda / \Delta \lambda$, see below), and their continua were not normalised, keeping the strong molecular absorption features from cool stars. Keeping the spectral shape also allowed relative flux calibration between the stars, by scaling the spectra to published Two Micron All Sky Survey (2MASS) photometry ( $J, H$ and $K_{\mathrm{s}} \mathrm{mag}$ ), implying that the integrated colours obtained from the spectra are consistent with those obtained by 2MASS.

The spectral types of the 210 library stars include F, G, K, M, $\mathrm{L}, \mathrm{S}$ and $\mathrm{C}$ types, with luminosity classes from supergiants (I) to dwarfs (V). The majority of the stars are cool stars, which dominate the light in the NIR. Around $60 \%$ of the stars are variable, including Cepheids, RR Lyrae and semi-regular variables. Additionally, some stars have two spectra, corrected and noncorrected for extinction. We kept these spectra as individual stars leaving us with a sample of 292 spectra.

irtfweb.ifa.hawaii.edu/ spex/IRTF_Spectral_Library/ 


\section{Determination of stellar parameters}

A crucial step towards using a spectral stellar library for SSP modelling (see Paper II) is to accurately know the atmospheric parameters of its stars to be able to tie them to the evolutionary tracks during the modelling. Moreover, the parametric coverage of the library is vital to understand the applicable ranges in the models constructed from its stars. For this purpose we apply two methods, one in which the $T_{\text {eff }}, \log g$ and metallicity of many of the stars in the IRTF spectral library are determined in a selfconsistent way from a sample of stars with well-determined parameters (see Sect. 3.1) and another in which we use the colourtemperature relations for different regimes (see Sect. 3.2). We use parameters from the literature to establish the validity of these methods.

\subsection{Full-spectrum fitting method}

Here we use the $U L y S S^{2}$ package (Koleva et al. 2009) to compare the IRTF spectra to a template library with known parameters, and to find the set of best-matching spectra. Briefly, ULySS fits a spectrum with a linear combination of non-linear components (in this case stellar spectra) convolved with a line-of-sight velocity distribution and multiplied by a polynomial continuum.

The parameters of the best-matching spectra along with their respective weights give the atmospheric parameters of the IRTF stars. Figure 2 shows examples of the fits for three stars (IRL003, IRL120 and IRL270) with their corresponding resulting parameters. It is important to mention that the full-spectrum fitting (FSF) approach used for measuring the atmospheric parameters was only used on a small region of the $K$-band (2.19 to $2.34 \mu \mathrm{m})$.

We choose as our primary template library 73 stars of the IRTF spectral library that are also found in the empirical stellar libraries MILES (Sánchez-Blázquez et al. 2006) and CaT (Cenarro et al. 2001). The parameters of those stars are determined using a compilation from the literature by Cenarro et al. (2007, hereafter C07). Additionally, we use as templates 52 stars observed in a section of the $K$-band (2.19 to $2.34 \mu \mathrm{m}$ ) by Mármol-Queraltó et al. (2008) which are also contained in the MILES and CaT libraries and for which there are also stellar atmospheric parameters available in $\mathrm{C} 07$.

For our analyses, we also create 108 interpolated stars whose parameters are selected from grids of different $T_{\text {eff }}, \log g$, and four metallicities that were within the limits provided by the empirical stars of our template library. Their temperature ranges from 2500 to $6500 \mathrm{~K}$, with steps of $500 \mathrm{~K}$ and their gravities range from -0.25 to $4.0 \mathrm{dex}$, with steps of $1.0 \mathrm{dex}$. The metallicities considered here are $-0.70,-0.4,0.0$ and 0.2 dex. The spectrum of each interpolated star is obtained by interpolating between empirical spectra of stars with known parameters in our template library. We apply an interpolation scheme similar to that of Vazdekis et al. (2003), which creates a box of stellar parameters around a given point with certain stellar parameters, i.e. the parameters of the star for which we want to obtain a spectrum. This box is flexible enough to expand until a sufficient number of adequate stars are found, if necessary, and is divided into eight cubes of different sizes that have the given point as a corner. This minimises the errors due to the lack of stars in certain regions of the distribution of stars. The size of the box is inversely proportional to the density of stars found

\footnotetext{
2 ulyss.univ-lyon $1 . \mathrm{fr}$
}
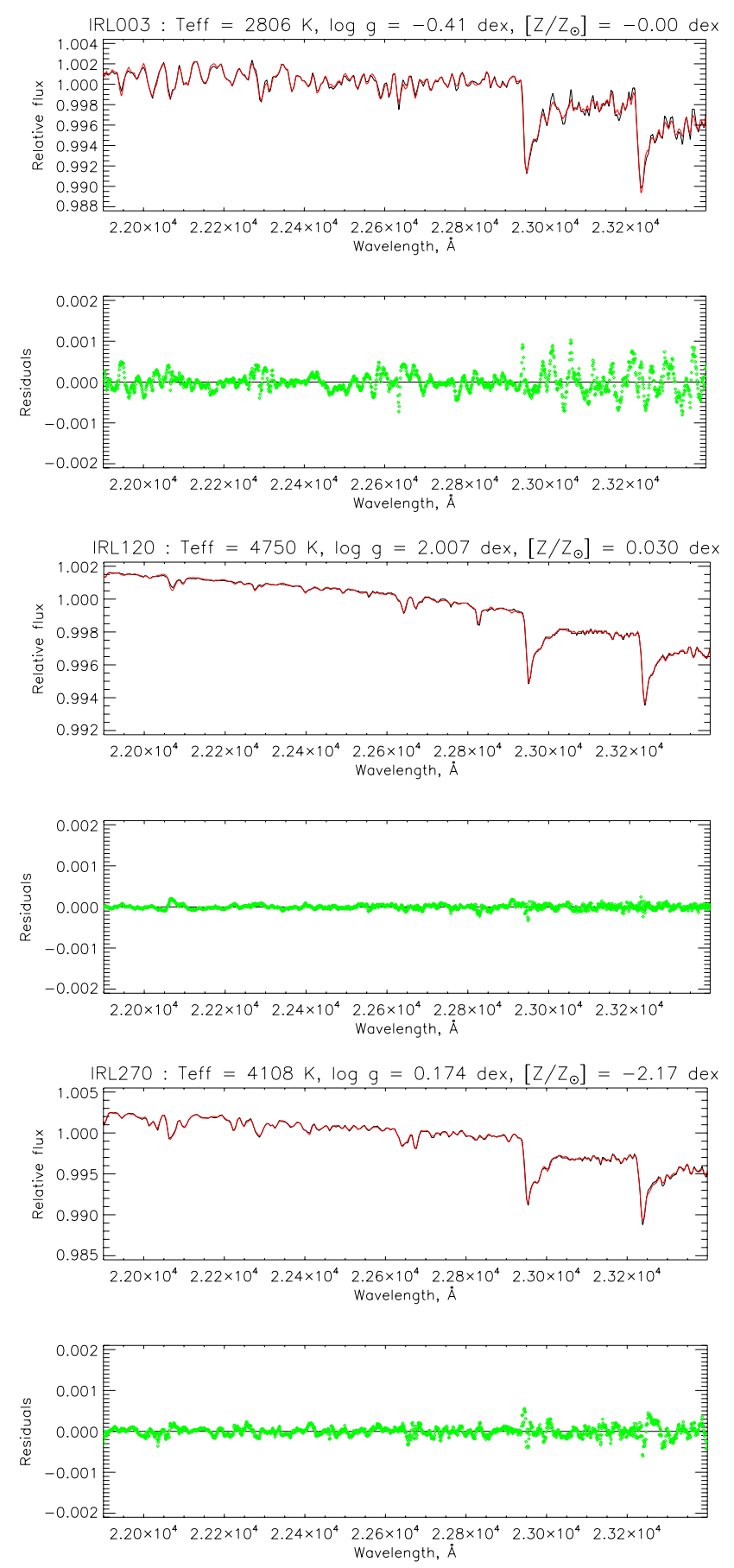

Fig. 2. Example of the results obtained with FSF. The name of the stars and the obtained parameters are given in the figure titles. In the upper panels, we show the observed spectrum (black lines) and the best fit model (red lines). In the lower panels, we show the residuals (green data points) which for these stars are less than $0.2 \%$. For details of the fits, see text.

around the point and the box can be as small as the typical uncertainties of the parameters of the template library. When the boxes are determined, the spectra of the stars that form each of the eight boxes are combined into eight different spectra. As a final step, these spectra are interpolated to obtain a final spectrum with the desired stellar parameters. This step is taken because the available interpolators with ULySS are only for optical wavelengths. We subsequently use the ULySS package, along 


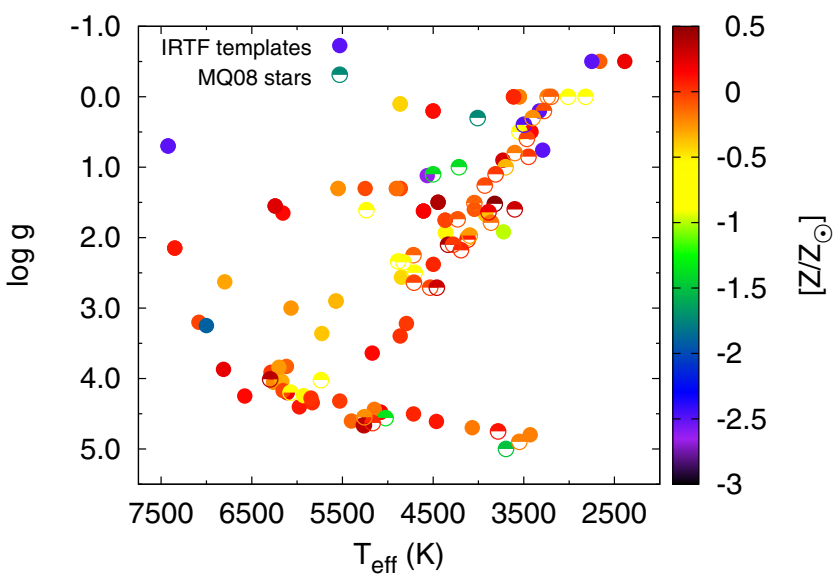

Fig. 3. HR diagram of the stars that compose the template library for the determination of stellar parameters in the IRTF spectral library. This template set is formed of 73 IRTF stars in common with the MILES and CaT empirical stellar libraries, and 52 additional stars observed by Mármol-Queraltó et al. (2008, MQ08).
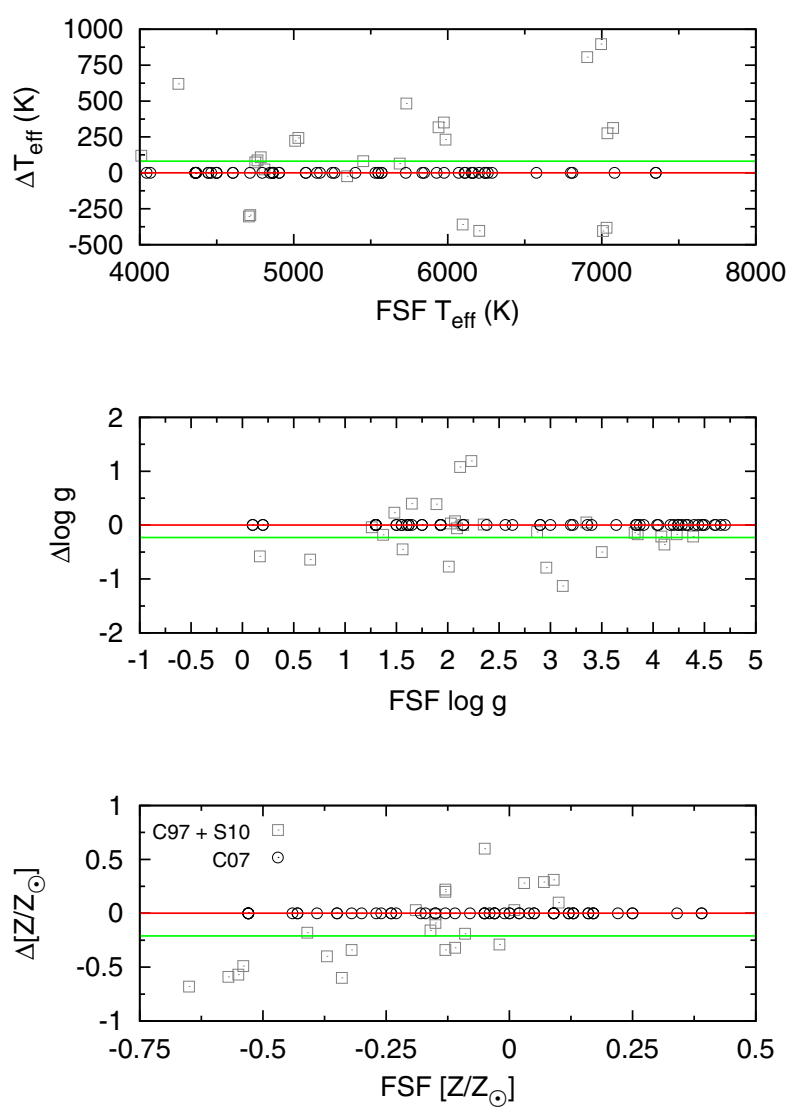

Fig. 4. Residuals from the comparison of the stellar parameters obtained from the FSF approach and the literature values from C07, S10 and C97 and the residuals (FSF - Lit). The red line is the one-to-one correlation. The green line marks the median of the differences ( $81 \mathrm{~K}$ for $T_{\text {eff }},-0.23$ for $\log g$ and -0.07 for metallicity) which represent the offset between non-C07 and C97 + S10 literature values.

with the 233 template stars described above to determine stellar atmospheric parameters in the full-spectrum approach.

We have run the FSF for the 73 IRTF-MILES stars (hereby C07-stars) not only to test this method but also to homogenise

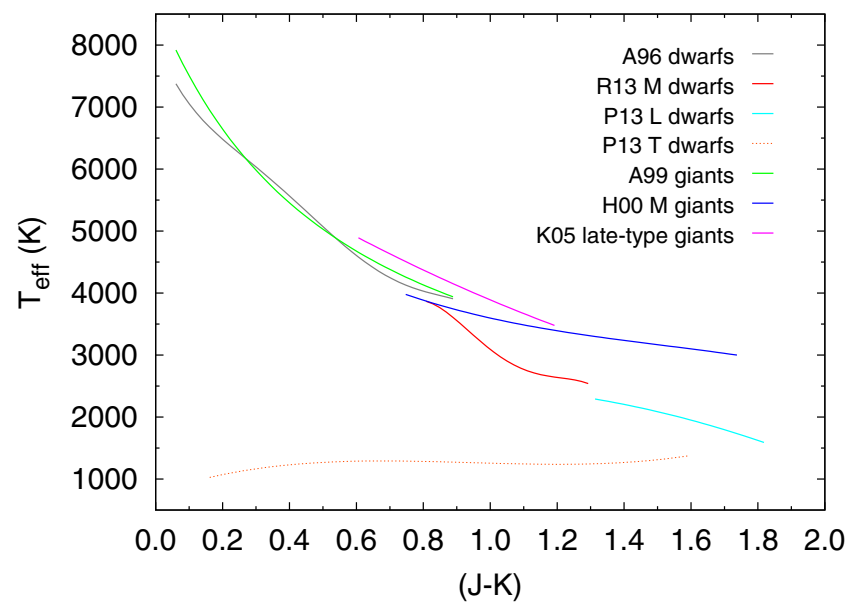

Fig. 5. Colour-temperature relations for different regimes of effective temperature and luminosity class from Alonso et al. (1996, A96), Rajpurohit et al. (2013, R13), Pecaut \& Mamajek (2013, P13), Alonso et al. (1999, A99), Houdashelt et al. (2000, H00) and Kučinskas et al. (2005, K05).

our "anchor" literature values. We have also applied the methodology to the IRTF stars with literature values from Soubiran et al. (2010, hereafter S10) and Cayrel de Strobel et al. (1997, hereafter C97), giving a combined sample of 40 stars hereby designated non-C07 stars.

Because of the lack of templates covering an adequate grid of atmospheric parameters for cool stars, the FSF method is not applied to stars with Teff below $4000 \mathrm{~K}$. Therefore, below $4000 \mathrm{~K}$ we adopt atmospheric parameters from the literature or the colour-temperature relation method (see Sect. 3.2).

Figure 4 shows that the parameters obtained for the nonC07 stars differ from the catalogues and therefore we calculate the offset between these values from the median of the difference $81 \mathrm{~K}$ for $T_{\text {eff }},-0.23$ for $\log g$ and -0.07 for metallicity. We apply the corresponding offset for each parameter to the literature values for the non-C07 stars in order to homogenise the "anchor" stellar atmospheric parameters in the C07 system.

From our analyses we see that most of the stars are in the metallicity range between $\approx-0.7$ and 0.2 , indicating that the IRTF spectral library is useful for NIR SP modelling mostly when working with populations near solar abundance.

\section{2. $T_{\text {eff }}$ and NIR-colour relations}

For this method, we determine the temperature as a function of NIR colours. For this purpose, we use colour-temperature relations (CTR) in the NIR, specifically $(J-K)$, for different regimes of effective temperatures and luminosity classes. For giants, we use the relations of Alonso et al. (1999, A99), Houdashelt et al. (2000, H00) and Kučinskas et al. (2005, K05) which are applied to F0-K5, M0-M7 and M8-cooler stars, respectively. For dwarfs, the relations of Alonso et al. (1996, A96), Rajpurohit et al. (2013, R13) and Pecaut \& Mamajek (2013, P13) are used for F0-K5, M0-M10 and L-T stars, respectively. We show these relations and their behaviour in Fig. 5.

Before applying these relations to those stars without literature parameters, we use them to re-calculate the values of the $\mathrm{C} 07$ and non-C07 stars in order to determine the difference between the literature values in the $\mathrm{C} 07$ system and colourtemperature relation results and anchor the latter values to the 
S. Meneses-Goytia et al.: Single stellar populations in the near-IR. I.

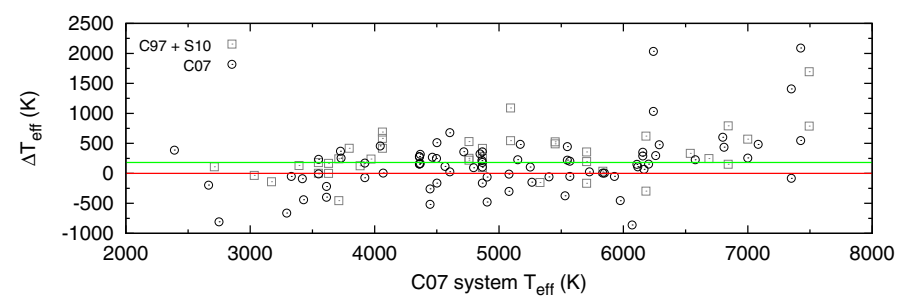

Fig. 6. Residuals from the comparison of the stellar parameters obtained from the colour-temperature relation (CTR) approach and the literature values from C07, S10 and C97 and the residual (Lit ${ }_{\text {C10 system }}-$ CTR). The red line is the one-to-one correlation. The green line marks the median of the difference $(181 \mathrm{~K})$ which represents the offset in temperatures between the CTR method and literature in the C07 system.

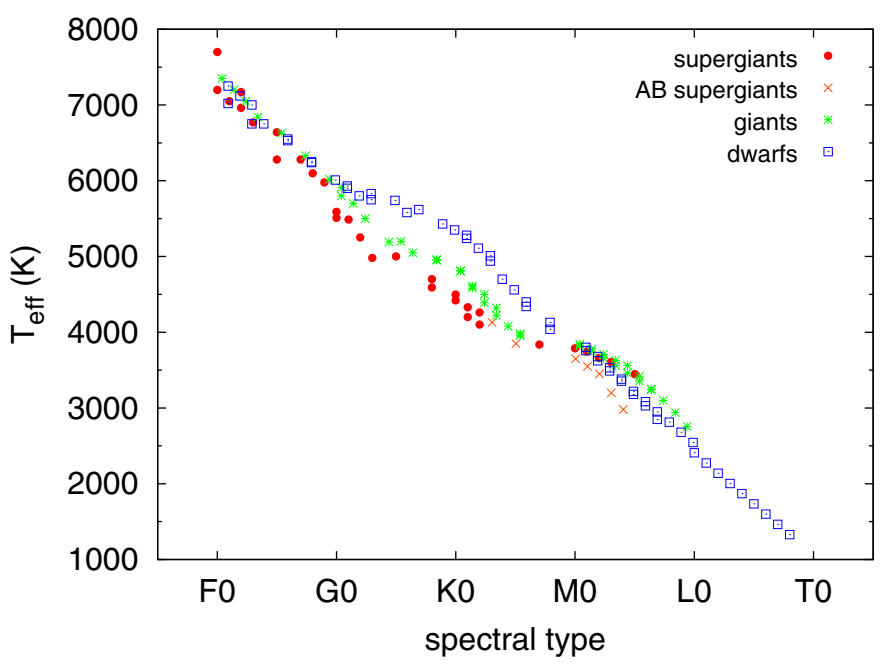

Fig. 7. Compilation of effective temperatures as a function of spectral type from Gray \& Corbally (2009) and Cox (1999).

former. This comparison and the respective median offset are shown in Fig. 6. This offset $(181 \mathrm{~K})$ is applied to the effective temperatures from the CTR approach. We note that the large difference between literature and CTR temperatures of very bright giants is due to the variable colours as a result of stellar pulsations.

\subsection{Selection of the atmospheric parameters}

To assess the accuracy of the parameters we obtained with both methods, we compare the parameters of the remaining 219 IRTF stars with literature values from the catalogues of S10 and C97. When literature data are not available, we infer the stellar temperature of the stars from their stellar classification by compiling information from Gray \& Corbally (2009) and Cox (1999), as shown in Fig. 7. To determine surface gravity, we use evolutionary tracks based on empirical stars as a function of temperature, gravity and spectral type from Straizys \& Kuriliene (1981). In Table A.1, we show our calculated parameters, including those from the literature, and those of the template stars.

The agreement between the measured parameters by the FSF method and the literature is reasonable (as shown in Figs. 8-10), with $\sigma_{T_{\text {eff }}}=366 \mathrm{~K}, \sigma_{\log g}=0.36 \mathrm{dex}$ and $\sigma_{\left[Z / Z_{\odot}\right]}=0.28 \mathrm{dex}$. This technique was limited by the parameter coverage of the

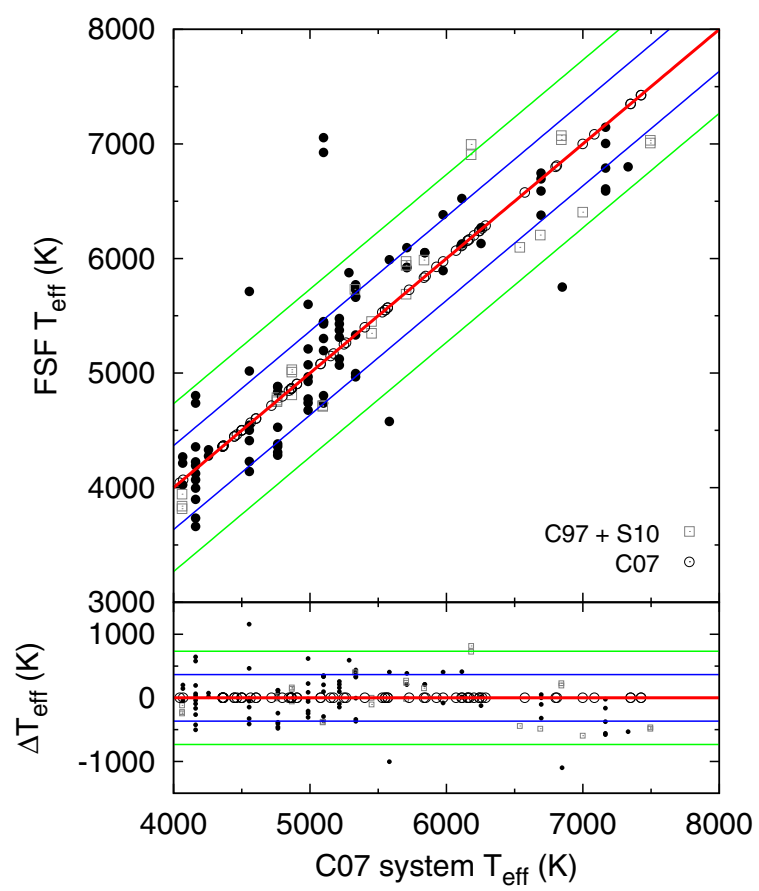

Fig. 8. Comparison of the effective temperature obtained from the FSF with literature values in the $\mathrm{C} 07$ system. The squares and circles are those stars with literature values from either C07, S10 or C97 already in the $\mathrm{C} 07$ system, for which parameters are computed using their stellar type. The black data points are stars that do not have literature values in C07, S10 or C97. The red line is the one-to-one relation. The blue and green lines mark the $1 \sigma$ and $2 \sigma$ confidence intervals, respectively. The

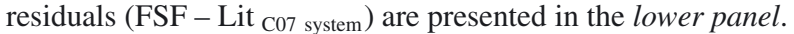

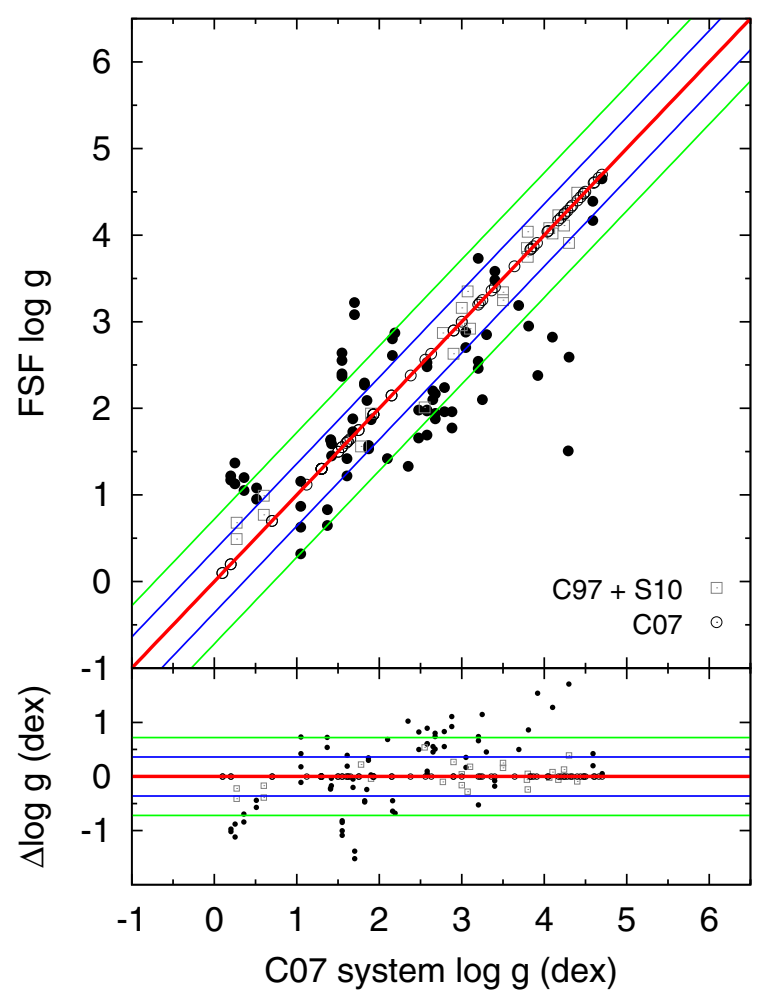

Fig. 9. As in Fig. 8 but for $\log g$.

template library which was dominated by solar metallicity giants and dwarfs between $\sim 3500$ and $7500 \mathrm{~K}$. The stars for which we 


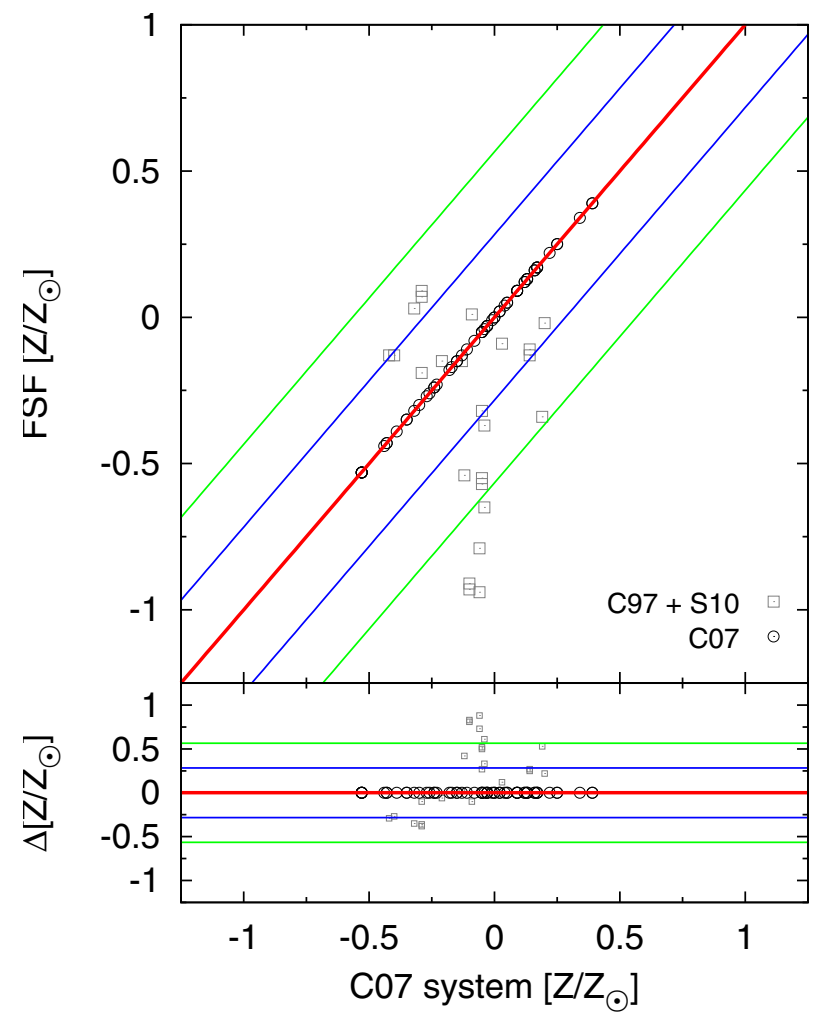

Fig. 10. As in Fig. 8 but for the stars with literature values $\left(\left[Z / Z_{\odot}\right]\right)$. We note that the diagram contains fewer stars than e.g. Fig. 9. This is because there are many stars without literature values, for which there is no way to determine their metallicity. For these stars we have assumed solar chemical abundances and have not plotted them here.

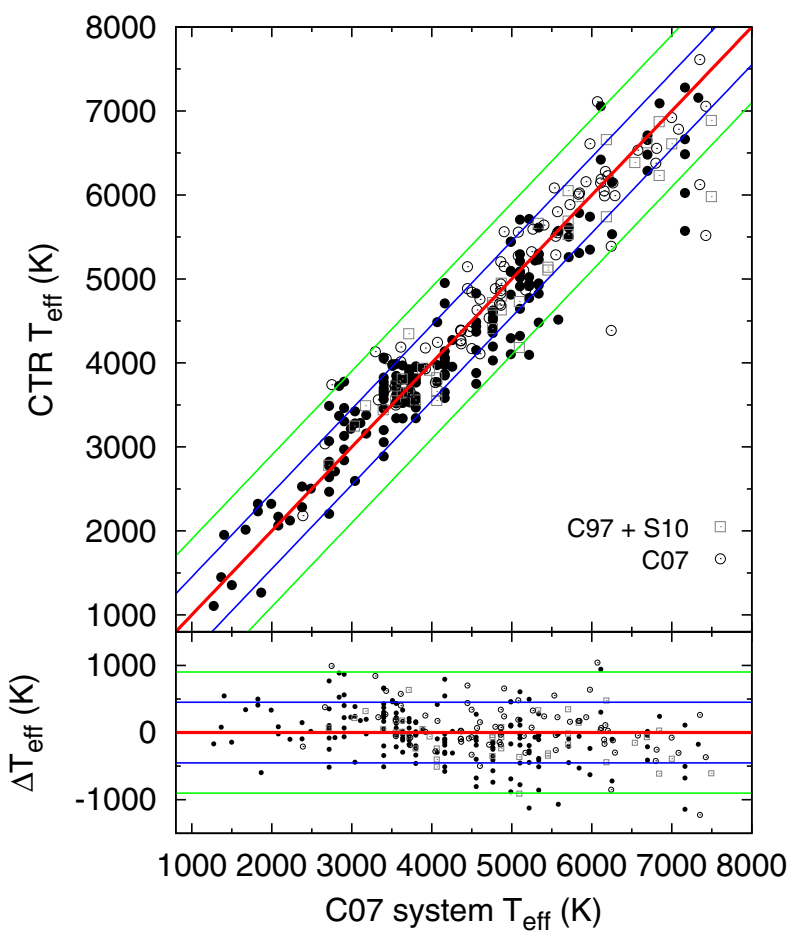

Fig. 11. As in Fig. 8 but here showing the effective temperature obtained from the $(J-K)-T_{\text {eff }}$ relations shown in Fig. 5.

could not determine good parameters are late-type giants (M6 and cooler) and M, L and T dwarfs $\left(T_{\text {eff }}<2500 \mathrm{~K}\right)$.

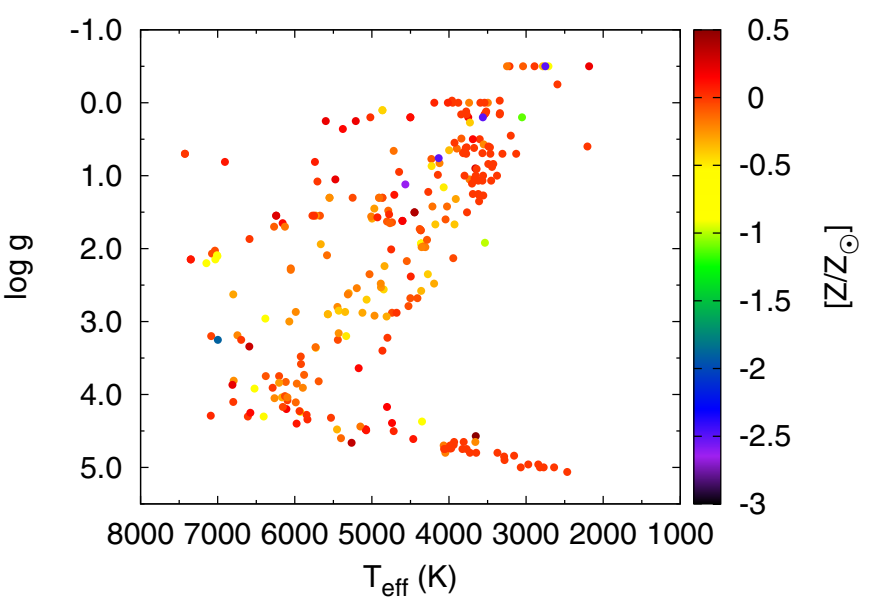

Fig. 12. Stellar atmospheric parameters for the stars of the IRTF spectral library. This shows the parameter coverage of this library for stellar population models. L- and T-type dwarfs were not included.

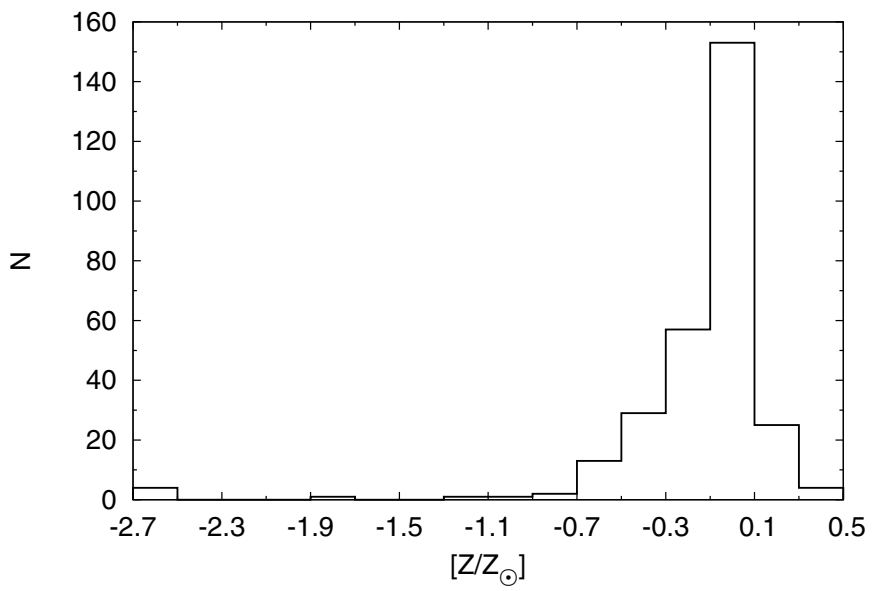

Fig. 13. Metallicity distribution function for the stars of the IRTF spectral library.

In Fig. 11 we show the resulting effective temperatures from the colour-temperature relations and the comparison with the literature values. This method also shows a good agreement with $\sigma_{T_{\text {eff }}}=451 \mathrm{~K}$, except for a few very bright giants whose colours oscillate due to stellar pulsations.

It is important to point out that these methods are independent from each other. The FSF determines the parameters of a given star by comparing its spectrum to an empirical spectral library whose stellar parameters were compiled from the literature (for details of this compilation see Cenarro et al. 2007). On the other hand, the CTR is based on the relation of observed photometry of stars and their effective temperature (see e.g. Alonso et al. 1999).

To compute the stellar population models (Paper II), we mainly use the parameters from the FSF and for those stars whose values are not in the $2 \sigma_{T_{\text {eff }}}$ confidence interval, we take the results from the colour-temperature relation that are also within the corresponding reliable limits. For $\log g$, we take the FSF results, or, if they are not within the $2 \sigma_{\log g}$ limits, we take the literature values. We prefer not to use the mass-luminosity relations to calculate the $\log g$ because it requires assigning a particular age and metallicity making the method subject to a 
S. Meneses-Goytia et al.: Single stellar populations in the near-IR. I.
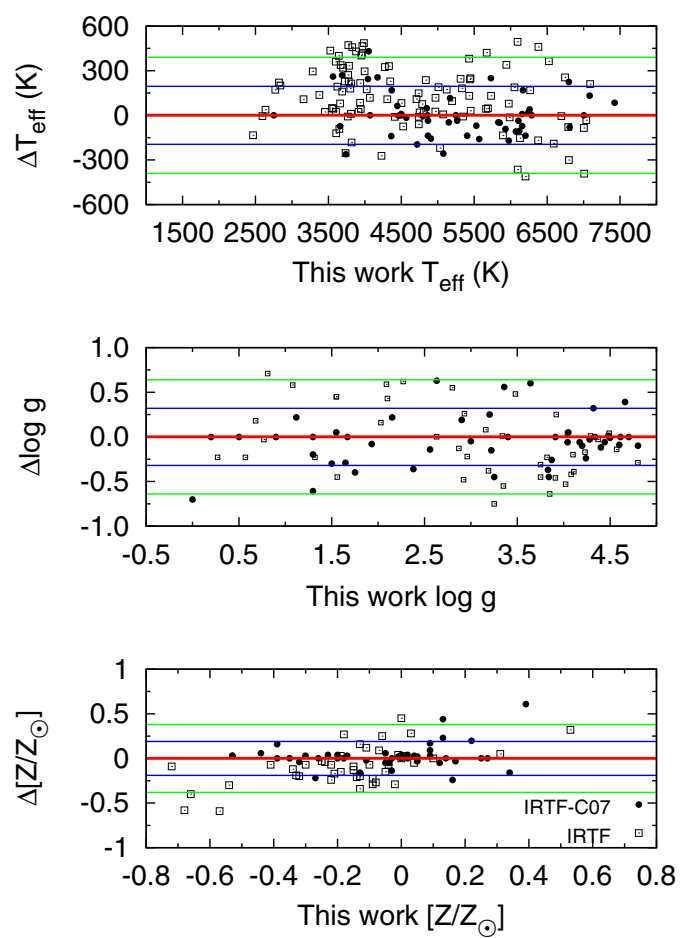

Fig. 14. Residuals from the comparison between the stellar atmospheric parameters for the stars in the IRTF spectral library and those compiled by Cesetti et al. (2013; present work - Cesetti). The circles represent the values for the IRTF stars with C07 stellar parameters and the squares the IRTF stars for which we determined their parameters. The red line is the one-to-one correlation. The blue and green lines mark the $1 \sigma$ and $2 \sigma$ confidence intervals, respectively.

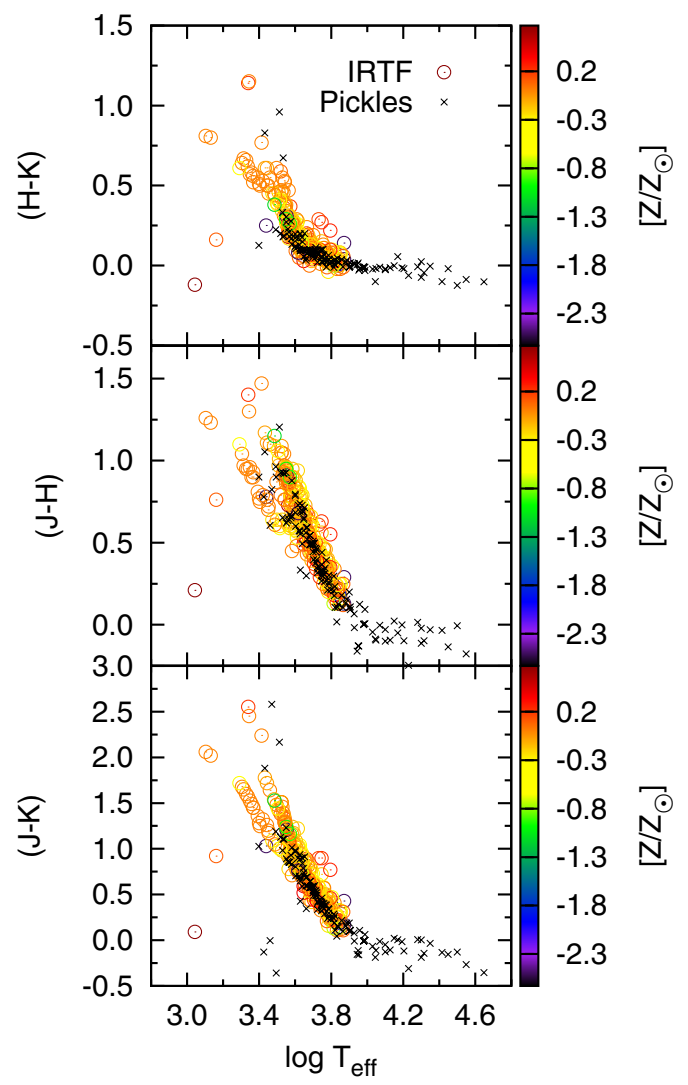

Fig. 15. Behaviour of the integrated colours of the IRTF spectral library stars as a function of effective temperature, compared with the Pickles stellar library (which is assumed to have solar metallicity).
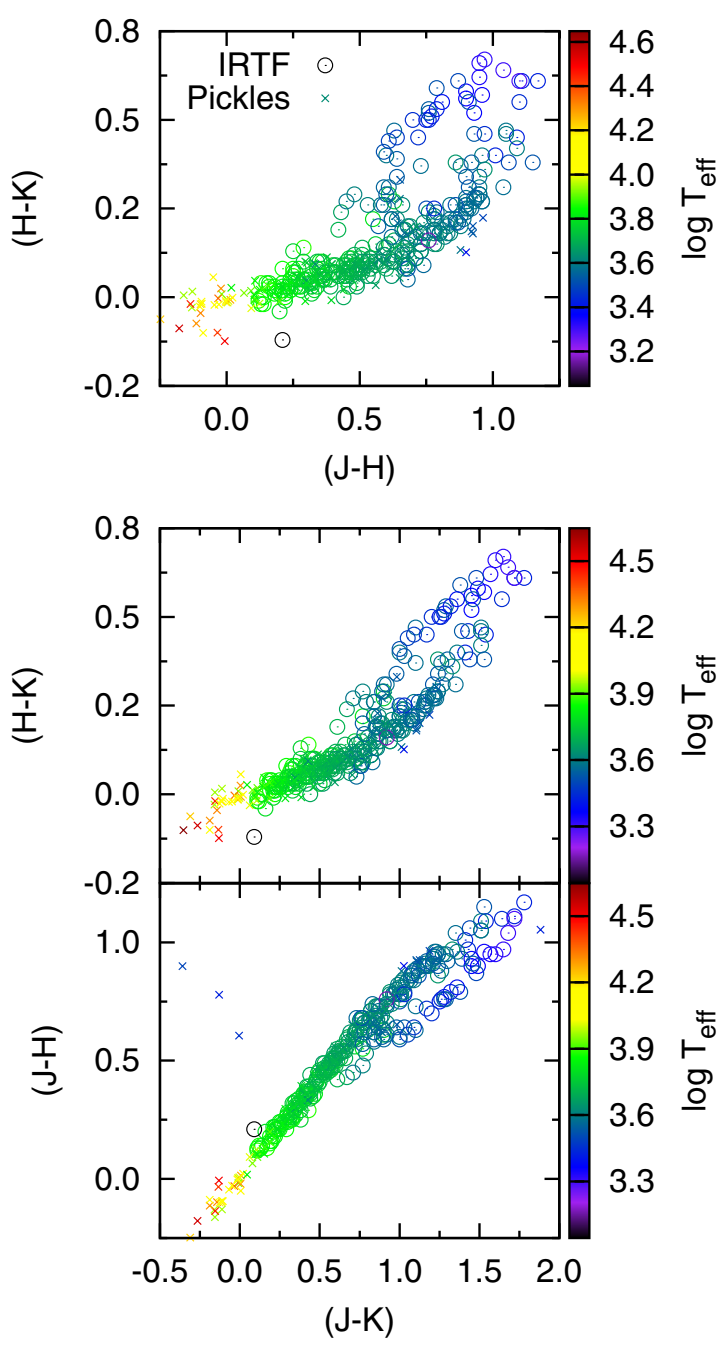

Fig. 16. Colour-colour diagrams of the stars in the IRTF spectral library, compared with the Pickles library (which is assumed to have solar metallicity).

bias. For outliers in metallicity, literature values were used if available, otherwise solar metallicity was chosen.

The calculated values within the $2 \sigma$ confidence intervals (for the respective parameter) are shown in Table A.1. Figure 12 shows the HR diagram of these stars and Fig. 13 their metallicity distribution function.

\subsection{Comparison with recent work}

We compare the atmospheric parameters of the IRTF spectral library stars with the survey of available stellar parameters compiled from the literature by Cesetti et al. (2013). In Fig. 14 we present the residuals of the comparisons for $T_{\text {eff }}$ (184 stars), $\log g$ (101 stars) and $\left[Z / Z_{\odot}\right]$ (95 stars). This comparison shows a reasonable agreement between our anchoring literature values from C07, our obtained ones and those from Cesetti et al. (2013), with $\sigma_{T_{\text {eff }}}=195 \mathrm{~K}, \sigma_{\log g}=0.32 \mathrm{dex}$ and $\sigma_{\left[Z / Z_{\odot}\right]}=0.19 \mathrm{dex}$.

\section{Flux calibration of the IRTF spectral library}

In this section, we test the flux calibration of the library and the behaviour of the stellar integrated colours as a function of 

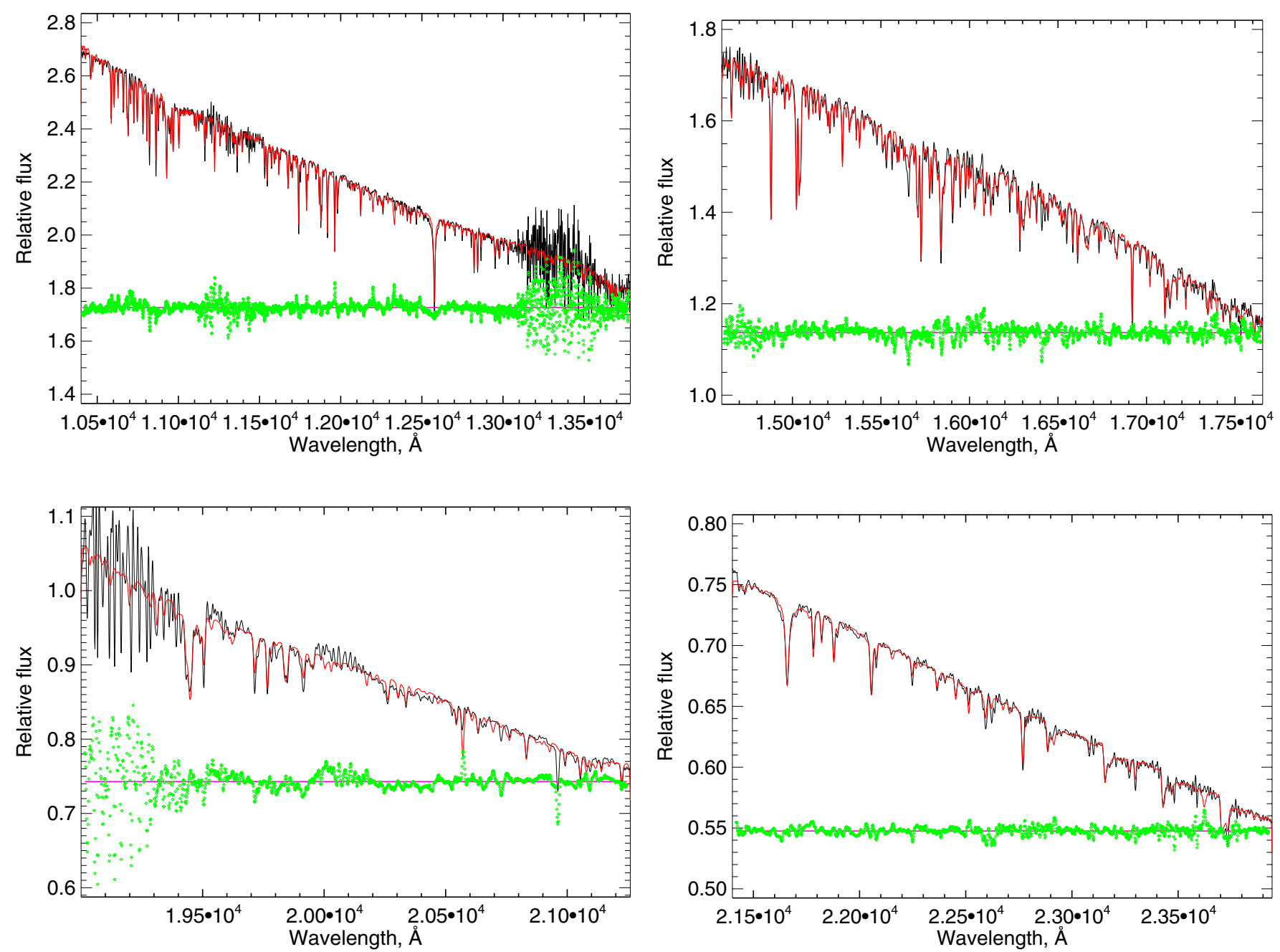

Fig. 17. Example of the FSF for a solar-like star IRL075. We show the observed spectrum (black lines), the best fit model (red lines) and the residuals (green data points). The poor fit in some regions is due to telluric absorption contamination.

the atmospheric parameters. The reliability of this calibration is important for the SP modelling when comparing to photometric observations of globular clusters and galaxies.

Relative $J, H$ and $K_{\mathrm{s}}$ fluxes were determined by integrating the spectral flux in these NIR bands using the Vega spectrum from Colina et al. (1996) as a zero-point. We use the response curves of the $J, H$ and $K$ filters of the Johnson-Cousins-Glass photometric system given by Bessell et al. (1998). We present in Fig. 15 a comparison of the colours with the library of Pickles (1998) for solar metallicity stars. We can see that our inferred parameters follow the same trend as those of Pickles. We draw the same conclusion from the colour-colour diagrams in Fig. 16.

\section{Determining the spectral resolution}

In order to asses the accuracy of our SSP models (Paper II), it is also important to characterise the spectral resolution of the stellar library to be used and to make sure that the radial velocities of all the stars have been set to zero. Therefore, to determine the nominal spectral resolution (FWHM) of the IRTF spectral library, we fit the stars with another template library of very high resolution.
We use the recent PHOENIX BT-Settl ${ }^{3}$ stellar models (Allard et al. 2012) as a template library. The stellar parameters of this theoretical library cover a wide range of temperature (2600-7000 K), luminosity class (from dwarfs to supergiants) and metallicity ( -0.5 to $0.5 \mathrm{dex})$. In the wavelength range that we use ( $J, H$ and $K$-bands), the full-width at half maximum (FWHM) of the library is $0.05 \AA$.

The FWHM of the IRTF spectral library has been determined by comparison with the BT-Settl library. Both libraries are rebinned to a velocity scale of $25 \mathrm{~km} \mathrm{~s}^{-1}$, after which the broadening of data and templates are measured using the penalised pixel-fitting method (pPXF, Cappellari \& Emsellem 2004). The templates are convolved with a Gaussian and fit to each individual test spectrum to determine the FWHM. To assess the dependence of resolution on wavelength, we divide the original datasets into four wavelength bins, representing the $J(1.04-1.44 \mu \mathrm{m})$ and $H(1.46-1.80 \mu \mathrm{m})$ bands, the atomic part of the $K$-band (1.90-2.14 $\mu \mathrm{m})$ and the molecular part of the $K$-band (2.14-2.41 $\mu \mathrm{m})$, with effective wavelengths of 1.22 , $1.62,2.02$ and $2.27 \mu \mathrm{m}$, respectively. The basic procedure is described in detail by Falcón-Barroso et al. (2011). Figure 17

\footnotetext{
3 phoenix.ens-lyon.fr/Grids/BT-Settl/AGSS2009/ SPECTRA/
} 

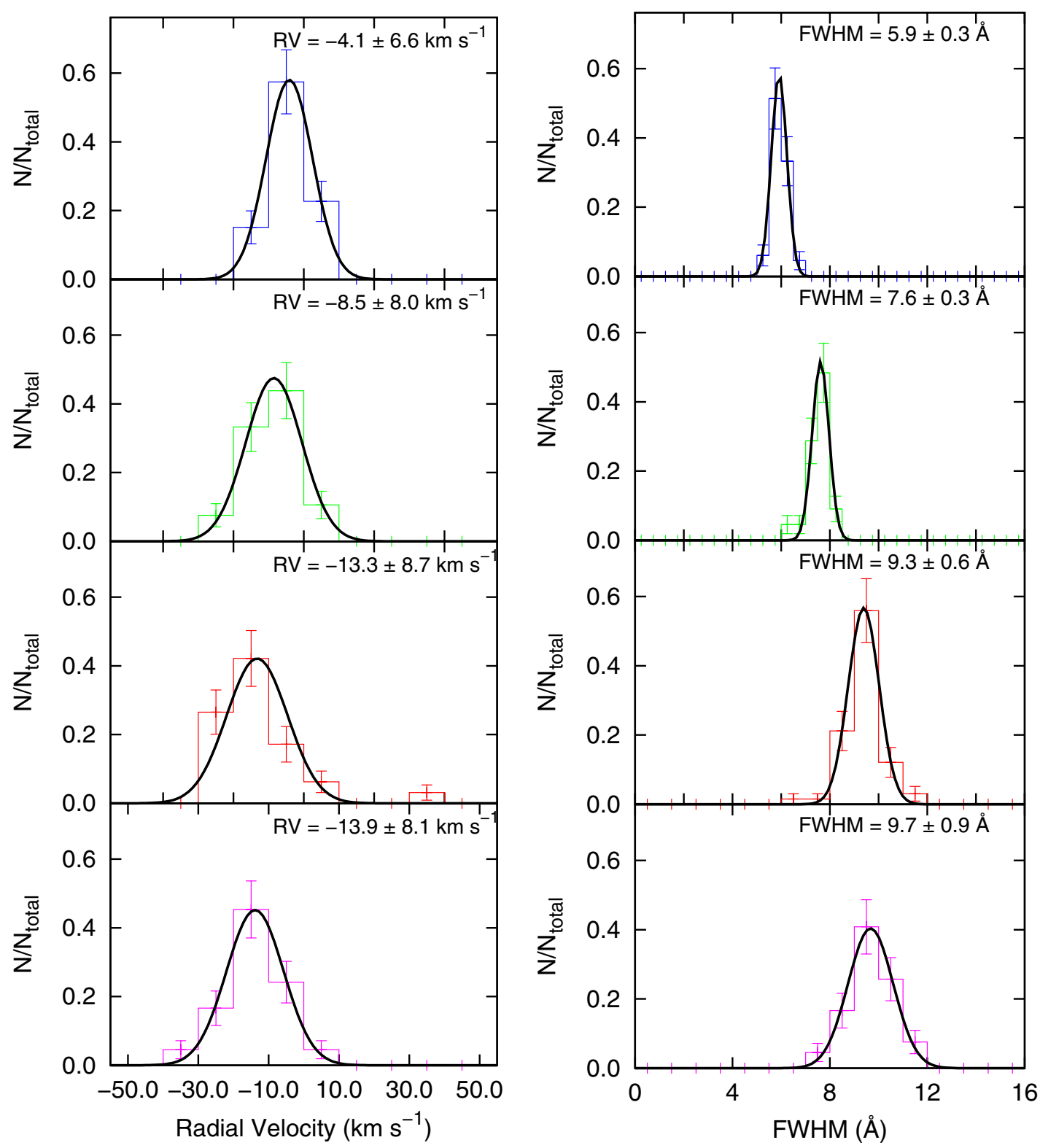

Fig. 18. Left panels: radial velocities of the IRTF spectral library as a function of wavelength. Right panel: FWHM as a function of wavelength. For the $J$-band, at $1.22 \mu \mathrm{m}$, the stars have an average FWHM of $5.9 \pm 0.3 \AA$. In the $H$-band, at $1.62 \mu$ m, the peak value is $7.6 \pm 0.3 \AA$. For the atomic-dominated part of the $K$-band, at $2.02 \mu \mathrm{m}$, the average resolution is $9.3 \pm 0.6 \AA$. And for the molecular-dominated part of the $K$-band, at $2.27 \mu \mathrm{m}$, the average FWHM is $9.7 \pm 0.9 \AA$.

shows an example of a solar-like star from the IRTF library and its fit using this procedure.

From this technique, we also obtain the radial velocity (RV) of the IRTF stars for each bin as shown in the left panel of Fig. 18. For the $J$-band, at $1.22 \mu \mathrm{m}$, most of stars have an RV of $-4.1 \pm 6.6 \mathrm{~km} \mathrm{~s}^{-1}$ (standard deviation). In the $H$-band, at $1.62 \AA$, the peak value is $-8.5 \pm 8.0 \mathrm{~km} \mathrm{~s}^{-1}$. For the atomic-dominated part of the $K$-band, at $2.02 \AA$, the radial velocity is $-13.3 \pm$ $8.7 \mathrm{~km} \mathrm{~s}^{-1}$, and for the molecular-dominated part of the $K$-band, at $2.27 \AA$, the $\mathrm{RV}$ is $-13.9 \pm 8.1 \mathrm{~km} \mathrm{~s}^{-1}$.

In Fig. 19 we show how the FWHM, the resolution $R$ and their respective scatters behave as a function of wavelength for the G stars in the library, in bin sizes of $1000 \AA$. As we see, the FWHM increases when going to the red.
The behaviour of the FWHM as a function of each wavelength bin is presented in the right panel histograms of Fig. 18. One can see that the resolution is not constant in wavelength, as is the case for the MILES library, but is approximately proportional with wavelength $(R$ constant), but with some additional variation as a function of wavelength. For the $J$-band, at $1.22 \mu \mathrm{m}$, most stars have an average FWHM of $5.9 \pm 0.3 \AA$. In the $H$-band, at $1.62 \AA$, the peak value is $7.6 \pm 0.3 \AA$. For the atomic-dominated part of the $K$-band, at $2.02 \AA$, the average resolution is $9.3 \pm 0.6 \AA$. And for the molecular-dominated part of the $K$-band, at $2.27 \AA$, the average FWHM is $9.7 \pm 0.9 \AA$. In terms of the spectral resolution, $R=2060 \pm 15,2163 \pm$ $16,2153 \pm 23$, and $2350 \pm 25$ at $1.22,1.62,2.02$, and $2.27 \mu \mathrm{m}$ respectively. 

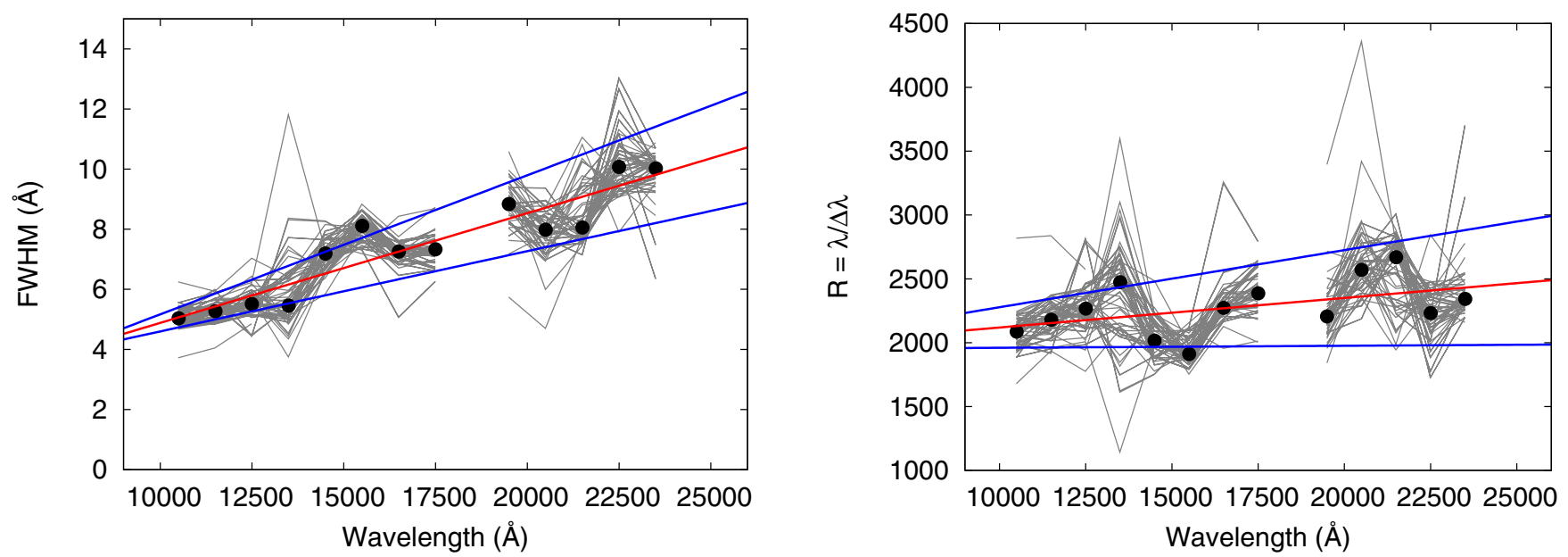

Fig. 19. Behaviour of the FWHM (left panel) and the resolving power $R$ (right panel) of the G stars of the IRTF spectral library (grey lines) as a function of wavelength. In both panels, the black points represent the mean values for those effective wavelengths and the blue lines mark the mean dispersion. In the left panel, the red line corresponds to a liner relation of the mean FWHM for each wavelength. In the right panel, the red line is a linear relation of the mean $R$ with effective wavelength.

These tests demonstrate that the resolution of this library is higher than most previous empirical libraries, such as Lançon \& Wood (2000).

\section{Final remarks}

We have studied in detail the accuracy and characteristics of the IRTF spectral library in the $J, H$ and $K$-bands. We have determined the parameter coverage of the IRTF spectral library, allowing us to understand the extent of its usefulness. We have also determined the accuracy of the flux calibration of the library. Additionally, we have measured the precise spectral resolution and radial velocity of the stars.

With these tests, we understand the possibilities and limitations of stellar population models using the IRTF spectral library as input (Paper II), over the $J, H$ and $K$-bands.

Cool late-type stars have a strong influence on the integrated flux in these wavelength regions. These stars are particularly relevant for studies of early-type galaxies. Since our library has cool stars, we can get a much better handle on the relative contribution of these stars in unresolved galaxies (Paper III).

Our rebinned IRTF spectral library spectra (i.e. with a constant dispersion in wavelength) and the resulting data of the analysis and characterisation are available online ${ }^{4}$.

Acknowledgements. The authors acknowledge the usage of the SIMBAD data base (operated at CDS, Strasbourg, France) and the IRTF spectral library database, and the referee. S.M.G. thanks T.J.L. de Boer and the Institute of Astronomy of the University of Cambridge for support during her visits. M.K. is a postdoctoral Marie Curie fellow (Grant PIEF-GA-2010-271780) and a fellow of the Fund for Scientific Research - Flanders, Belgium (FWO11/PDO/147). J.F.B. and A.V. acknowledge the support by the Programa Nacional del Astronomía y Astrofísica of the Spanish Ministry of Science and Innovation undergrant AYA2013-48226-C3-1-P, as well as from the FP7 Marie Curie Actions of the European Commission, via the Initial Training Network DAGAL under REA grant agreement number 289313.

\section{Appendix A: The stars composing the IRTF spectral library and their atmospheric parameters.}

In this appendix, we compile the available information for the IRTF stars. The first column shows the ID numbers of the IRTF stars, corresponding to the order of stars adopted in Rayner et al. (2009), Cushing et al. (2005). Indented IDs indicate stars that have not been corrected for extinction. The second column lists the names given by the IRTF library database. The coordinates of each star are given in the third and fourth columns. The fifth column gives the stellar class of each star according to the IRTF library. The fifth, sixth and seventh columns present the atmospheric parameters of each star, determined from either the full-spectrum fitting method $\left({ }^{\mathrm{FSF}}\right)$, the colour-temperature rela-

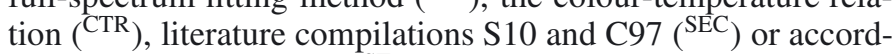
ing to their stellar type $\left({ }^{\mathrm{ST}}\right)$. For stars from the Cenarro et al. (2007) catalogue (labelled ${ }^{\mathrm{C} 07}$ ) the atmospheric parameters are determined using the full-spectrum fitting method and recovered as the same values given in the literature. Finally, the eight, ninth and tenth columns list the NIR colours of each star as calculated from the spectrum (see Sect. 4).

\footnotetext{
4 smg.astro-research.net/
} 
S. Meneses-Goytia et al.: Single stellar populations in the near-IR. I.

Table A.1. Reference relation for the IRTF spectral library stars.

\begin{tabular}{|c|c|c|c|c|c|c|c|c|c|c|}
\hline ID & Star & RA & Dec & Class & $T_{\text {eff }}(\mathrm{K})$ & $\log g$ & {$\left[Z / Z_{\odot}\right]$} & $(J-H)$ & $(J-K)$ & $(H-K)$ \\
\hline IRL280 & BRI B0021-0214 & 002424.63 & -015820.14 & M9.5V & $2466^{\mathrm{CTR}}$ & $5.06^{\mathrm{ST}}$ & $0.00^{\mathrm{ST}}$ & 0.81 & 1.36 & 0.55 \\
\hline IRL147 & HD 002901 & 003247.52 & +540711.81 & K2III & $4410^{\mathrm{FSF}}$ & $2.68^{\mathrm{ST}}$ & $-0.07^{\mathrm{FSF}}$ & 0.67 & 0.75 & 0.08 \\
\hline IRL194 & 2MASS J00361617+1821104 & 003616.17 & +1821 10.47 & L3.5 & $2324^{\mathrm{CTR}}$ & $\geq 5.0^{\mathrm{ST}}$ & $0.00^{\mathrm{ST}}$ & 0.90 & 1.45 & 0.56 \\
\hline IRL181 & HD 003346 & 003646.44 & +442918.91 & K6IIIa & $3943^{\mathrm{FSF}}$ & $2.13^{\mathrm{ST}}$ & $-0.05^{\mathrm{FSF}}$ & 0.84 & 1.05 & 0.21 \\
\hline IRL072 & HD 003421 & 003721.21 & +352358.20 & $\mathrm{G} 2 \mathrm{Ib}$ & $5663^{\mathrm{FSF}}$ & $1.94^{\mathrm{FSF}}$ & $-0.33^{\mathrm{FSF}}$ & 0.42 & 0.48 & 0.06 \\
\hline IRL153 & HD 003765 & 004049.26 & +4011 13.83 & $\mathrm{~K} 2 \mathrm{~V}$ & $5073^{\mathrm{FSF}}$ & $4.49^{\mathrm{FSF}}$ & $0.10^{\mathrm{FSF}}$ & 0.46 & 0.53 & 0.07 \\
\hline IRL252 & HD 004408 & 004632.95 & +152831.81 & M4III & $3566^{\mathrm{CTR}}$ & $1.07^{\mathrm{ST}}$ & $0.00^{\mathrm{ST}}$ & 0.93 & 1.21 & 0.27 \\
\hline IRL260 & Gl 051 & 010319.72 & +62 2155.70 & M5V & $3649^{\mathrm{CTR}}$ & $4.8^{\mathrm{ST}}$ & $0.00^{\mathrm{ST}}$ & 0.61 & 0.92 & 0.31 \\
\hline IRL014 & HD 006130 & 010337.00 & +610429.36 & F0II & $7031^{\mathrm{FSF}}$ & $2.15^{\mathrm{FSF}}$ & $-0.55^{\mathrm{FSF}}$ & 0.25 & 0.33 & 0.08 \\
\hline IRL013 & HD 006130 ext & 010337.00 & +610429.36 & FOII & $7008^{\mathrm{FSF}}$ & $2.09^{\mathrm{FSF}}$ & $-0.57^{\mathrm{FSF}}$ & 0.16 & 0.19 & 0.03 \\
\hline IRL277 & IRAS 01037+1219 & 010625.98 & +123553.00 & M8III & $3777^{\mathrm{CTR}}$ & $0.7^{\mathrm{ST}}$ & $0.00^{\mathrm{ST}}$ & 2.50 & 4.21 & 1.71 \\
\hline IRL082 & HD 006474 ext & 010659.74 & +634623.38 & G4Ia & $6241^{\mathrm{C} 07}$ & $1.55^{\mathrm{C} 07}$ & $0.25^{\mathrm{C} 07}$ & 0.35 & 0.46 & 0.10 \\
\hline IRL083 & HD 006474 & 010659.74 & +634623.38 & G4Ia & $6241^{\mathrm{C} 07}$ & $1.55^{\mathrm{C} 07}$ & $0.25^{\mathrm{C} 07}$ & 0.55 & 0.77 & 0.22 \\
\hline IRL055 & HD 006903 & 010949.20 & +193930.26 & F9IIIa & $5570^{\mathrm{C} 07}$ & $2.9^{\mathrm{C} 07}$ & $-0.35^{\mathrm{C} 07}$ & 0.36 & 0.42 & 0.06 \\
\hline IRL054 & HD 006903 ext & 010949.20 & +193930.26 & F9IIIa & $5570^{\mathrm{C} 07}$ & $2.9^{\mathrm{C} 07}$ & $-0.35^{\mathrm{C} 07}$ & 0.32 & 0.37 & 0.04 \\
\hline IRL202 & HD 236697 & 011953.61 & +581830.73 & M0.5Ib & $3565^{\mathrm{CTR}}$ & $0.69^{\mathrm{ST}}$ & $0.00^{\mathrm{ST}}$ & 0.93 & 1.21 & 0.28 \\
\hline IRL201 & HD $236697 \mathrm{ext}$ & 011953.61 & +581830.73 & M0.5Ib & $3814^{\mathrm{CTR}}$ & $0.69^{\mathrm{ST}}$ & $0.00^{\mathrm{ST}}$ & 0.78 & 0.97 & 0.19 \\
\hline IRL010 & HD 007927 & 012004.91 & +581353.80 & F0Ia & $7425^{\mathrm{C} 07}$ & $0.7^{\mathrm{C} 07}$ & $0.00^{\mathrm{C} 07}$ & 0.29 & 0.43 & 0.14 \\
\hline IRL009 & HD 007927 ext & 012004.91 & +581353.80 & F0Ia & $7425^{\mathrm{C} 07}$ & $0.7^{\mathrm{C} 07}$ & $0.00^{\mathrm{C} 07}$ & 0.13 & 0.17 & 0.04 \\
\hline IRL209 & $\mathrm{BD}+60265 \mathrm{ext}$ & 013333.05 & +613330.73 & M1.5Ib & $3771^{\mathrm{CTR}}$ & $0.61^{\mathrm{ST}}$ & $0.00^{\mathrm{ST}}$ & 0.76 & 1.01 & 0.24 \\
\hline IRL210 & $\mathrm{BD}+60265$ & 013333.05 & +613330.73 & M1.5Ib & $3468^{\mathrm{CTR}}$ & $0.61^{\mathrm{ST}}$ & $0.00^{\mathrm{ST}}$ & 0.97 & 1.33 & 0.36 \\
\hline IRL124 & HD 009852 & 013751.23 & +615141.74 & K0.5III & $4678^{\mathrm{FSF}}$ & $2.88^{\mathrm{ST}}$ & $0.01^{\mathrm{FSF}}$ & 0.67 & 0.81 & 0.14 \\
\hline IRL123 & HD 009852 ext & 013751.23 & +615141.74 & K0.5III & $4740^{\mathrm{FSF}}$ & $2.88^{\mathrm{ST}}$ & $-0.05^{\mathrm{FSF}}$ & 0.55 & 0.61 & 0.06 \\
\hline IRL067 & HD 010307 & 014147.14 & +423648.12 & G1V & $5847^{\mathrm{C} 07}$ & $4.28^{\mathrm{C} 07}$ & $0.02^{\mathrm{C} 07}$ & 0.32 & 0.34 & 0.03 \\
\hline IRL144 & HD 010476 & 014229.76 & +201606.60 & K1V & $5150^{\mathrm{C} 07}$ & $4.44^{\mathrm{C} 07}$ & $-0.17^{\mathrm{C} 07}$ & 0.45 & 0.53 & 0.08 \\
\hline IRL225 & HD 010465 ext & 014311.10 & +483100.36 & M2Ib & $3783^{\mathrm{CTR}}$ & $0.62^{\mathrm{ST}}$ & $0.00^{\mathrm{ST}}$ & 0.78 & 1.00 & 0.22 \\
\hline IRL226 & HD 010465 & 014311.10 & +483100.36 & M2Ib & $3673^{\mathrm{CTR}}$ & $0.62^{\mathrm{ST}}$ & $0.00^{\mathrm{ST}}$ & 0.84 & 1.09 & 0.26 \\
\hline IRL081 & HD 010697 & 014455.82 & +200459.33 & G3Va & $5688^{\mathrm{FSF}}$ & $3.82^{\mathrm{FSF}}$ & $-0.09^{\mathrm{FSF}}$ & 0.36 & 0.44 & 0.08 \\
\hline IRL039 & HD 011443 & 015304.90 & +2934 43.78 & F6IV & $6288^{\mathrm{C} 07}$ & $3.91^{\mathrm{C} 07}$ & $0.00^{\mathrm{C} 07}$ & 0.27 & 0.35 & 0.08 \\
\hline IRL189 & 2MASS J02081833+2542533 & 020818.33 & +254253.30 & $\mathrm{~L} 1$ & $2285^{\mathrm{CTR}}$ & $\geq 5.0^{\mathrm{ST}}$ & $0.00^{\mathrm{ST}}$ & 0.90 & 1.49 & 0.58 \\
\hline IRL016 & HD 013174 & 020925.33 & +255623.59 & FOIII & $7000^{\mathrm{C} 07}$ & $3.25^{\mathrm{C} 07}$ & $-1.90^{\mathrm{C} 07}$ & 0.18 & 0.19 & 0.01 \\
\hline IRL257 & HD 014386 & 021920.79 & -025839.49 & M5III & $3668^{\mathrm{CTR}}$ & $1.04^{\mathrm{ST}}$ & $0.00^{\mathrm{ST}}$ & 0.63 & 1.10 & 0.47 \\
\hline IRL212 & HD 014404 ext & 022142.41 & +575146.12 & M1Iab & $3952^{\text {CTR }}$ & $0.0^{\mathrm{SEC}}$ & $0.00^{\mathrm{ST}}$ & 0.71 & 0.87 & 0.17 \\
\hline IRL213 & HD 014404 & 022142.41 & +575146.12 & M1Iab & $3593^{\mathrm{CTR}}$ & $0.0^{\mathrm{SEC}}$ & $0.00^{\mathrm{ST}}$ & 0.90 & 1.18 & 0.28 \\
\hline IRL242 & HD 014469 ext & 022206.89 & +563614.86 & M3Iab & $3773^{\mathrm{CTR}}$ & $0.16^{\mathrm{ST}}$ & $0.00^{\mathrm{ST}}$ & 0.75 & 1.00 & 0.25 \\
\hline IRL243 & HD 014469 & 022206.89 & +563614.86 & M3Iab & $3538^{\mathrm{CTR}}$ & $0.16^{\mathrm{ST}}$ & $0.00^{\mathrm{ST}}$ & 0.90 & 1.24 & 0.34 \\
\hline IRL232 & HD 014488 ext & 022224.29 & +5706 34.36 & M3.5Iab & $3525^{\text {CTR }}$ & $0.14^{\mathrm{ST}}$ & $0.00^{\mathrm{ST}}$ & 0.88 & 1.26 & 0.37 \\
\hline IRL233 & HD 014488 & 022224.29 & +570634.36 & M3.5Iab & $3340^{\text {CTR }}$ & $0.14^{\mathrm{ST}}$ & $0.00^{\mathrm{ST}}$ & 1.05 & 1.51 & 0.47 \\
\hline IRL101 & HD 016139 ext & 023617.99 & +272820.36 & G7.5IIIa & $5123^{\mathrm{FSF}}$ & $2.88^{\mathrm{FSF}}$ & $-0.31^{\mathrm{FSF}}$ & 0.47 & 0.57 & 0.10 \\
\hline IRL102 & HD 016139 & 023617.99 & +272820.36 & G7.5IIIa & $5069^{\mathrm{FSF}}$ & $2.7 \mathrm{FSF}$ & $-0.41^{\mathrm{FSF}}$ & 0.51 & 0.63 & 0.12 \\
\hline IRL166 & HD 016068 & 023652.80 & +555455.41 & K3II & $4285^{\mathrm{FSF}}$ & $1.88^{\mathrm{FSF}}$ & $-0.09^{\mathrm{FSF}}$ & 0.78 & 0.94 & 0.15 \\
\hline IRL165 & HD 016068 ext & 023652.80 & +555455.41 & K3II & $4380^{\mathrm{FSF}}$ & $1.73^{\mathrm{FSF}}$ & $-0.06^{\mathrm{FSF}}$ & 0.62 & 0.69 & 0.06 \\
\hline IRL029 & HD 016232 & 023657.74 & +243853.02 & $\mathrm{~F} 4 \mathrm{~V}$ & $6097^{\mathrm{FSF}}$ & $4.08^{\mathrm{FSF}}$ & $-0.02^{\mathrm{FSF}}$ & 0.23 & 0.26 & 0.03 \\
\hline IRL034 & HD 017918 & 025311.69 & +162900.45 & F5III & $6695^{\mathrm{FSF}}$ & $3.25^{\mathrm{FSF}}$ & $0.00^{\mathrm{ST}}$ & 0.22 & 0.22 & 0.01 \\
\hline IRL200 & DENIS-P J0255.0-4700 & 025503.57 & -470050.99 & L8 & $1952^{\mathrm{CTR}}$ & $\geq 5.0^{\mathrm{ST}}$ & $0.00^{\mathrm{ST}}$ & 1.10 & 1.72 & 0.61 \\
\hline IRL266 & HD 018191 & 025548.49 & +18 1953.90 & M6III & $3536^{\mathrm{CTR}}$ & $1.92^{\mathrm{C} 07}$ & $-0.99^{\mathrm{C} 07}$ & 0.95 & 1.24 & 0.29 \\
\hline IRL092 & HD 018474 & 025949.79 & +471314.48 & G5III & $5878^{\mathrm{FSF}}$ & $3.73^{\mathrm{FSF}}$ & $-0.11^{\mathrm{FSF}}$ & 0.46 & 0.50 & 0.03 \\
\hline IRL249 & HD 019058 & 030510.59 & +385024.99 & M4IIIa & $3549^{\mathrm{CTR}}$ & $0.57^{\mathrm{SEC}}$ & $-0.22^{\mathrm{SEC}}$ & 0.96 & 1.23 & 0.27 \\
\hline IRL205 & HD 019305 & 030626.73 & +015754.63 & MOV & $3934^{\mathrm{CTR}}$ & $4.65^{\mathrm{ST}}$ & $0.00^{\mathrm{ST}}$ & 0.69 & 0.85 & 0.16 \\
\hline IRL061 & HD 020619 & 031901.89 & -025035.49 & $\mathrm{G} 1.5 \mathrm{~V}$ & $6094^{\mathrm{FSF}}$ & $4.04^{\mathrm{FSF}}$ & $-0.18^{\mathrm{FSF}}$ & 0.38 & 0.45 & 0.07 \\
\hline IRL109 & HD 020618 & 031955.79 & +270416.06 & G7IV & $5430^{\mathrm{FSF}}$ & $3.16^{\mathrm{FSF}}$ & $-0.25^{\mathrm{FSF}}$ & 0.49 & 0.57 & 0.09 \\
\hline IRL279 & LP 412-31 & 032059.65 & +185423.31 & M8V & $2841^{\mathrm{CTR}}$ & $4.96^{\mathrm{ST}}$ & $0.00^{\mathrm{ST}}$ & 0.72 & 1.17 & 0.45 \\
\hline IRL063 & HD 021018 ext & 032338.98 & +045255.57 & G1III & $5924^{\mathrm{FSF}}$ & $3.48^{\mathrm{FSF}}$ & $-0.04^{\mathrm{FSF}}$ & 0.35 & 0.41 & 0.06 \\
\hline IRL064 & HD 021018 & 032338.98 & +045255.57 & G1III & $5920^{\mathrm{FSF}}$ & $3.58^{\mathrm{FSF}}$ & $-0.04^{\mathrm{FSF}}$ & 0.40 & 0.49 & 0.09 \\
\hline IRL028 & HD 021770 & 033226.26 & +460324.69 & F4III & $6204^{\mathrm{FSF}}$ & $3.75^{\mathrm{ST}}$ & $-0.04^{\mathrm{SEC}}$ & 0.21 & 0.23 & 0.02 \\
\hline IRL286 & LP 944-20 & 033935.22 & -352544.09 & M9V & $2821^{\mathrm{CTR}}$ & $5.0^{\mathrm{ST}}$ & $0.00^{\mathrm{ST}}$ & 0.70 & 1.20 & 0.50 \\
\hline IRL145 & HD 023082 ext & 034405.77 & +445304.91 & $\mathrm{K} 2.5 \mathrm{II}$ & $4270^{\mathrm{FSF}}$ & $1.22^{\mathrm{FSF}}$ & $0.00^{\mathrm{FSF}}$ & 0.63 & 0.73 & 0.10 \\
\hline IRL146 & HD 023082 & 034405.77 & +445304.91 & $\mathrm{K} 2.5 \mathrm{II}$ & $4215^{\mathrm{FSF}}$ & $1.42^{\mathrm{FSF}}$ & $-0.16^{\mathrm{FSF}}$ & 0.78 & 0.96 & 0.18 \\
\hline IRL227 & HD 023475 ext & 034931.27 & +653133.50 & M2II & $3800^{\mathrm{CTR}}$ & $1.0^{\mathrm{ST}}$ & $0.00^{\mathrm{ST}}$ & 0.80 & 0.98 & 0.18 \\
\hline
\end{tabular}


Table A.1. continued.

\begin{tabular}{|c|c|c|c|c|c|c|c|c|c|c|}
\hline ID & Star & RA & Dec & Class & $T_{\text {eff }}(\mathrm{K})$ & $\log g$ & {$\left[Z / Z_{\odot}\right]$} & $(J-H)$ & $(J-K)$ & $(H-K)$ \\
\hline IRL228 & HD 023475 & 034931.27 & +653133.50 & M2II & $3650^{\mathrm{CTR}}$ & $1.0^{\mathrm{ST}}$ & $0.00^{\mathrm{ST}}$ & 0.88 & 1.12 & 0.23 \\
\hline IRL027 & HD 026015 & 040741.98 & +150946.02 & F3V & $7090^{\mathrm{CTR}}$ & $4.29^{\mathrm{ST}}$ & $0.04^{\mathrm{SEC}}$ & 0.14 & 0.12 & -0.02 \\
\hline IRL141 & HD 025975 & 040815.38 & +3743 38.98 & K1III & $4966^{\mathrm{FSF}}$ & $2.92^{\mathrm{FSF}}$ & $-0.24^{\mathrm{FSF}}$ & 0.47 & 0.53 & 0.06 \\
\hline IRL106 & HD 025877 & 040927.57 & +595429.05 & G7II & $4904^{\mathrm{C} 07}$ & $1.3^{\mathrm{C} 07}$ & $-0.15^{\mathrm{C} 07}$ & 0.45 & 0.52 & 0.06 \\
\hline IRL105 & HD 025877 ext & 040927.57 & +595429.05 & G7II & $4904^{\mathrm{C} 07}$ & $1.3^{\mathrm{C} 07}$ & $-0.15^{\mathrm{C} 07}$ & 0.39 & 0.42 & 0.03 \\
\hline IRL052 & HD 027383 & 041954.85 & +163121.32 & F8V & $6127^{\mathrm{FSF}}$ & $4.02^{\mathrm{FSF}}$ & $-0.05^{\mathrm{FSF}}$ & 0.24 & 0.25 & 0.02 \\
\hline IRL017 & HD 027397 & 041957.70 & +140206.71 & FOIV & $6799^{\mathrm{FSF}}$ & $4.1^{\mathrm{ST}}$ & $0.00^{\mathrm{ST}}$ & 0.13 & 0.11 & -0.02 \\
\hline IRL251 & HD 027598 & 042041.34 & -164947.91 & M4III & $3623^{\mathrm{CTR}}$ & $1.07^{\mathrm{ST}}$ & $0.00^{\mathrm{ST}}$ & 0.90 & 1.15 & 0.25 \\
\hline IRL100 & HD 027277 & 042053.54 & +501618.20 & G6III & $5196^{\mathrm{FSF}}$ & $2.54^{\mathrm{FSF}}$ & $-0.20^{\mathrm{FSF}}$ & 0.47 & 0.55 & 0.08 \\
\hline IRL037 & HD 027524 & 042131.64 & +210223.56 & F5V & $6576^{\mathrm{C} 07}$ & $4.25^{\mathrm{C} 07}$ & $0.13^{\mathrm{C} 07}$ & 0.21 & 0.23 & 0.02 \\
\hline IRL235 & HD 028487 & 042938.94 & +050951.35 & M3.5III & $3553^{\mathrm{CTR}}$ & $1.27^{\mathrm{ST}}$ & $0.00^{\mathrm{ST}}$ & 0.96 & 1.22 & 0.27 \\
\hline IRL234 & HD 028487 ext & 042938.94 & +050951.35 & M3.5III & $3603^{\mathrm{CTR}}$ & $1.27^{\mathrm{ST}}$ & $0.00^{\mathrm{ST}}$ & 0.92 & 1.17 & 0.25 \\
\hline IRL001 & HD 031996 & 045936.34 & -144822.53 & C7.6 & $2181^{\mathrm{CTR}}$ & $-0.5^{\mathrm{C} 07}$ & $0.20^{\mathrm{C} 07}$ & 1.40 & 2.55 & 1.14 \\
\hline IRL207 & HD 035601 ext & 052710.21 & +295515.79 & M1.5Iab & $3738^{\mathrm{CTR}}$ & $0.0^{\mathrm{C} 07}$ & $-0.20^{\mathrm{C} 07}$ & 0.79 & 1.03 & 0.24 \\
\hline IRL208 & HD 035601 & 052710.21 & +295515.79 & M1.5Iab & $3497^{\mathrm{CTR}}$ & $0.0^{\mathrm{C} 07}$ & $-0.20^{\mathrm{C} 07}$ & 0.95 & 1.29 & 0.34 \\
\hline IRL159 & HD 035620 ext & 052738.88 & +342833.21 & K3III & $4367^{\mathrm{C} 07}$ & $1.75^{\mathrm{C} 07}$ & $-0.03^{\mathrm{C} 07}$ & 0.64 & 0.77 & 0.13 \\
\hline IRL160 & HD 035620 & 052738.88 & +342833.21 & K3III & $4367^{\mathrm{C} 07}$ & $1.75^{\mathrm{C} 07}$ & $-0.03^{\mathrm{C} 07}$ & 0.68 & 0.84 & 0.15 \\
\hline IRL180 & HD 036003 & 052826.09 & -032958.39 & K5V & $4464^{\mathrm{C} 07}$ & $4.61^{\mathrm{C} 07}$ & $0.09^{\mathrm{C} 07}$ & 0.61 & 0.72 & 0.11 \\
\hline IRL140 & HD 036134 & 052923.68 & -032647.01 & K1III & $4837^{\mathrm{FSF}}$ & $2.24^{\mathrm{FSF}}$ & $-0.32^{\mathrm{FSF}}$ & 0.60 & 0.68 & 0.08 \\
\hline IRL211 & HD 036395 & 053127.39 & -034038.03 & $\mathrm{M} 1.5 \mathrm{~V}$ & $3651^{\mathrm{CTR}}$ & $4.57^{\mathrm{SEC}}$ & $0.53^{\mathrm{SEC}}$ & 0.73 & 0.92 & 0.18 \\
\hline IRL197 & SDSS J053951.99-005902.0 & 053952.00 & -005901.90 & L5 & $2325^{\mathrm{CTR}}$ & $\geq 5.0^{\mathrm{ST}}$ & $0.00^{\mathrm{ST}}$ & 0.93 & 1.45 & 0.52 \\
\hline IRL253 & Gl 213 & 054209.26 & +122921.62 & M4V & $4063^{\text {CTR }}$ & $4.75^{\mathrm{ST}}$ & $0.00^{\mathrm{ST}}$ & 0.53 & 0.79 & 0.26 \\
\hline IRL240 & HD 039045 ext & 055125.74 & +320728.89 & M3III & $3690^{\mathrm{CTR}}$ & $1.25^{\mathrm{ST}}$ & $0.00^{\mathrm{ST}}$ & 0.86 & 1.08 & 0.22 \\
\hline IRL241 & HD 039045 & 055125.74 & +320728.89 & M3III & $3619^{\mathrm{CTR}}$ & $1.25^{\mathrm{ST}}$ & $0.00^{\mathrm{ST}}$ & 0.90 & 1.15 & 0.24 \\
\hline IRL217 & HD 039801 ext & 055510.30 & +072425.43 & M2Ia & $4190^{\mathrm{CTR}}$ & $0.0^{\mathrm{C} 07}$ & $0.05^{\mathrm{C} 07}$ & 0.68 & 0.73 & 0.05 \\
\hline IRL218 & HD 039801 & 055510.30 & +072425.43 & M2Ia & $4016^{\mathrm{CTR}}$ & $0.0^{\mathrm{C} 07}$ & $0.05^{\mathrm{C} 07}$ & 0.74 & 0.83 & 0.09 \\
\hline IRL068 & HD 039949 ext & 055705.55 & +271859.95 & $\mathrm{G} 2 \mathrm{Ib}$ & $5671^{\mathrm{FSF}}$ & $1.55^{\mathrm{ST}}$ & $-0.07^{\mathrm{FSF}}$ & 0.41 & 0.48 & 0.07 \\
\hline IRL069 & HD 039949 & 055705.55 & +271859.95 & $\mathrm{G} 2 \mathrm{Ib}$ & $5726^{\mathrm{FSF}}$ & $1.55^{\mathrm{ST}}$ & $-0.25^{\mathrm{FSF}}$ & 0.47 & 0.58 & 0.11 \\
\hline IRL025 & HD 040535 & 055901.08 & -092256.00 & F2III & $6791^{\mathrm{FSF}}$ & $3.81^{\mathrm{ST}}$ & $-0.24^{\mathrm{FSF}}$ & 0.21 & 0.25 & 0.04 \\
\hline IRL292 & 2MASS J05591915-1404489 & 055919.14 & -140448.88 & $\mathrm{~T} 4.5$ & $1105^{\mathrm{CTR}}$ & $\geq 5.0^{\mathrm{ST}}$ & $0.67^{\mathrm{SEC}}$ & 0.21 & 0.09 & -0.12 \\
\hline IRL239 & HD 040239 & 055956.09 & +455612.24 & M3IIb & $3558^{\mathrm{CTR}}$ & $1.0^{\mathrm{ST}}$ & $0.00^{\mathrm{ST}}$ & 0.94 & 1.22 & 0.28 \\
\hline IRL219 & HD 042581 & 061034.61 & -215152.71 & M1V & $3937^{\text {CTR }}$ & $4.69^{\mathrm{ST}}$ & $0.00^{\mathrm{ST}}$ & 0.66 & 0.85 & 0.19 \\
\hline IRL070 & HD 042454 ext & 061205.48 & +292931.72 & $\mathrm{G} 2 \mathrm{Ib}$ & $5772^{\mathrm{FSF}}$ & $1.55^{\mathrm{ST}}$ & $-0.01^{\mathrm{FSF}}$ & 0.36 & 0.40 & 0.05 \\
\hline IRL071 & HD 042454 & 061205.48 & +292931.72 & $\mathrm{G} 2 \mathrm{Ib}$ & $5751^{\mathrm{FSF}}$ & $1.55^{\mathrm{ST}}$ & $0.02^{\mathrm{FSF}}$ & 0.46 & 0.57 & 0.11 \\
\hline IRL290 & HD 044544 & 062223.85 & +032527.88 & SC5.5 & $3055^{\mathrm{CTR}}$ & $0.2^{\mathrm{ST}}$ & $-1.12^{\mathrm{SEC}}$ & 1.15 & 1.53 & 0.38 \\
\hline IRL127 & HD 044391 ext & 062247.87 & +275912.03 & $\mathrm{~K} 0 \mathrm{Ib}$ & $4786^{\mathrm{FSF}}$ & $1.48^{\mathrm{FSF}}$ & $-0.13^{\mathrm{FSF}}$ & 0.54 & 0.66 & 0.11 \\
\hline IRL128 & HD 044391 & 062247.87 & +275912.03 & $\mathrm{~K} 0 \mathrm{Ib}$ & $4764^{\mathrm{FSF}}$ & $1.65^{\mathrm{FSF}}$ & $-0.11^{\mathrm{FSF}}$ & 0.61 & 0.76 & 0.15 \\
\hline IRL006 & HD 044984 & 062528.17 & +144319.16 & $\mathrm{CN} 4$ & $3248^{\mathrm{CTR}}$ & $-0.5^{\mathrm{ST}}$ & $-0.17^{\mathrm{SEC}}$ & 1.01 & 1.41 & 0.40 \\
\hline IRL174 & HD 045977 ext & 063007.30 & -114832.19 & $\mathrm{~K} 4 \mathrm{~V}$ & $4738^{\mathrm{FSF}}$ & $4.39^{\mathrm{FSF}}$ & $0.14^{\mathrm{FSF}}$ & 0.49 & 0.56 & 0.07 \\
\hline IRL175 & HD 045977 & 063007.30 & -114832.19 & $\mathrm{~K} 4 \mathrm{~V}$ & $4805^{\mathrm{FSF}}$ & $4.17^{\mathrm{FSF}}$ & $0.15^{\mathrm{FSF}}$ & 0.53 & 0.62 & 0.09 \\
\hline IRL007 & HD 048664 & 064440.71 & +031858.65 & CN5 & $2710^{\mathrm{CTR}}$ & $-0.5^{\mathrm{ST}}$ & $-0.62^{\mathrm{SEC}}$ & 1.17 & 1.78 & 0.61 \\
\hline IRL048 & HD 051956 & 065931.74 & +005500.36 & F8Ib & $6130^{\mathrm{FSF}}$ & $1.7^{\mathrm{ST}}$ & $-0.17^{\mathrm{FSF}}$ & 0.35 & 0.42 & 0.07 \\
\hline IRL047 & HD 051956 ext & 065931.74 & +005500.36 & F8Ib & $6268^{\mathrm{FSF}}$ & $1.7^{\mathrm{ST}}$ & $-0.10^{\mathrm{FSF}}$ & 0.27 & 0.30 & 0.03 \\
\hline IRL 248 & Gl 268 & 071001.83 & +383146.06 & $\mathrm{M} 4.5 \mathrm{~V}$ & $3772^{\mathrm{CTR}}$ & $4.75^{\mathrm{ST}}$ & $0.00^{\mathrm{ST}}$ & 0.60 & 0.89 & 0.29 \\
\hline IRL003 & HD 057160 & 072059.00 & +245958.07 & CJ5 & $2889^{\mathrm{CTR}}$ & $-0.5^{\mathrm{ST}}$ & $0.00^{\mathrm{ST}}$ & 1.10 & 1.64 & 0.55 \\
\hline IRL099 & HD 058367 & 072538.89 & +091633.95 & G6IIb & $5009^{\mathrm{FSF}}$ & $1.56^{\mathrm{FSF}}$ & $-0.19^{\mathrm{FSF}}$ & 0.49 & 0.57 & 0.09 \\
\hline IRL236 & G1 273 & 072724.49 & +051332.83 & $\mathrm{M} 3.5 \mathrm{~V}$ & $3971^{\mathrm{CTR}}$ & $4.74^{\mathrm{ST}}$ & $0.00^{\mathrm{ST}}$ & 0.58 & 0.84 & 0.26 \\
\hline IRL237 & CD-314916 ext & 074102.62 & -314059.10 & M3Iab & $3778^{\mathrm{CTR}}$ & $0.12^{\mathrm{ST}}$ & $0.00^{\mathrm{ST}}$ & 0.78 & 1.00 & 0.22 \\
\hline IRL238 & CD-314916 & 074102.62 & -314059.10 & M3Iab & $3517^{\mathrm{CTR}}$ & $0.12^{\mathrm{ST}}$ & $0.00^{\mathrm{ST}}$ & 0.95 & 1.27 & 0.31 \\
\hline IRL289 & HD 062164 & 074217.46 & -105247.18 & S5 & $3198^{\mathrm{CTR}}$ & $0.45^{\mathrm{ST}}$ & $0.00^{\mathrm{ST}}$ & 1.06 & 1.44 & 0.38 \\
\hline IRL188 & 2MASS J07464256+2000321 & 074642.56 & +200032.18 & L0.5 & $2504^{\mathrm{CTR}}$ & $\geq 5.0^{\mathrm{ST}}$ & $0.00^{\mathrm{ST}}$ & 0.77 & 1.28 & 0.51 \\
\hline IRL137 & HD 063302 ext & 074738.52 & -155926.48 & K1Ia & $4500^{\mathrm{C} 07}$ & $0.2^{\mathrm{C} 07}$ & $0.12^{\mathrm{C} 07}$ & 0.51 & 0.60 & 0.10 \\
\hline IRL138 & HD 063302 & 074738.52 & -155926.48 & K1Ia & $4500^{\mathrm{C} 07}$ & $0.2^{\mathrm{C} 07}$ & $0.12^{\mathrm{C} 07}$ & 0.67 & 0.86 & 0.19 \\
\hline IRL288 & HD 064332 & 075305.27 & -113729.35 & S4.5 & $3727^{\mathrm{CTR}}$ & $0.27^{\mathrm{SEC}}$ & $-0.41^{\mathrm{SEC}}$ & 0.90 & 1.16 & 0.26 \\
\hline IRL255 & Gl 299 & 081157.57 & +084622.05 & M4V & $4055^{\mathrm{CTR}}$ & $4.75^{\mathrm{ST}}$ & $0.00^{\mathrm{ST}}$ & 0.48 & 0.77 & 0.29 \\
\hline IRL254 & G1 299 ext & 081157.57 & +084622.05 & M4V & $3828^{\mathrm{CTR}}$ & $4.75^{\mathrm{ST}}$ & $0.00^{\mathrm{ST}}$ & 0.45 & 0.71 & 0.27 \\
\hline IRL265 & HD 069243 & 081633.82 & +114334.45 & M6III & $3377^{\mathrm{CTR}}$ & $1.0^{\mathrm{ST}}$ & $0.00^{\mathrm{ST}}$ & 0.91 & 1.46 & 0.55 \\
\hline IRL002 & HD 070138 & 081943.09 & -181552.84 & CJ4.5IIIa & $3216^{\mathrm{CTR}}$ & $-0.5^{\mathrm{ST}}$ & $0.00^{\mathrm{ST}}$ & 0.97 & 1.43 & 0.46 \\
\hline IRL199 & 2MASS J08251968+2115521 & 082519.68 & +211552.12 & L7.5 & $1355^{\mathrm{CTR}}$ & $\geq 5.0^{\mathrm{ST}}$ & $0.00^{\mathrm{ST}}$ & 1.23 & 2.02 & 0.80 \\
\hline
\end{tabular}


S. Meneses-Goytia et al.: Single stellar populations in the near-IR. I.

Table A.1. continued.

\begin{tabular}{|c|c|c|c|c|c|c|c|c|c|c|}
\hline ID & Star & RA & Dec & Class & $T_{\text {eff }}(\mathrm{K})$ & $\log g$ & {$\left[Z / Z_{\odot}\right]$} & $(J-H)$ & $(J-K)$ & $(H-K)$ \\
\hline IRL264 & GJ 1111 & 082949.34 & +264633.74 & M6.5V & $3285^{\mathrm{CTR}}$ & $4.85^{\mathrm{ST}}$ & $0.00^{\mathrm{ST}}$ & 0.60 & 1.00 & 0.40 \\
\hline IRL062 & HD 074395 & 084340.37 & -071401.43 & $\mathrm{G} 1 \mathrm{Ib}$ & $5250^{\mathrm{C} 07}$ & $1.3^{\mathrm{C} 07}$ & $-0.05^{\mathrm{C} 07}$ & 0.40 & 0.47 & 0.07 \\
\hline IRL031 & HD 075555 & 085221.79 & +445351.46 & F5.5III & $6745^{\mathrm{FSF}}$ & $3.19^{\mathrm{FSF}}$ & $-0.22^{\mathrm{FSF}}$ & 0.24 & 0.28 & 0.04 \\
\hline IRL116 & HD 075732 ext & 085235.81 & +281950.95 & G8V & $5079^{\mathrm{C} 07}$ & $4.48^{\mathrm{C} 07}$ & $0.16^{\mathrm{C} 07}$ & 0.37 & 0.44 & 0.07 \\
\hline IRL117 & HD 075732 & 085235.81 & +281950.95 & G8V & $5079^{\mathrm{C} 07}$ & $4.48^{\mathrm{C} 07}$ & $0.16^{\mathrm{C} 07}$ & 0.41 & 0.50 & 0.09 \\
\hline IRL284 & LH S2065 & 085336.19 & -032932.11 & M9V & $2638^{\mathrm{CTR}}$ & $5.0^{\mathrm{ST}}$ & $0.00^{\mathrm{ST}}$ & 0.79 & 1.32 & 0.53 \\
\hline IRL075 & HD 076151 & 085417.94 & -052604.05 & $\mathrm{G} 2 \mathrm{~V}$ & $5940^{\mathrm{FSF}}$ & $4.23^{\mathrm{FSF}}$ & $0.01^{\mathrm{FSF}}$ & 0.34 & 0.41 & 0.08 \\
\hline IRL005 & HD 076221 & 085522.88 & +171352.58 & CN4.5 & $2782^{\mathrm{CTR}}$ & $-0.5^{\mathrm{ST}}$ & $-0.37^{\mathrm{SEC}}$ & 1.11 & 1.72 & 0.61 \\
\hline IRL008 & HD 076846 & 085948.94 & +334626.47 & CR2IIIa & $5582^{S T}$ & $2.09^{\mathrm{FSF}}$ & $-0.15^{\mathrm{FSF}}$ & 0.56 & 0.72 & 0.15 \\
\hline IRL030 & HD 087822 & 100815.88 & +313614.58 & F4V & $6377^{\mathrm{FSF}}$ & $3.75^{\mathrm{FSF}}$ & $-0.08^{\mathrm{FSF}}$ & 0.21 & 0.24 & 0.03 \\
\hline IRL221 & Gl 381 & 101204.69 & -024105.07 & $\mathrm{M} 2.5 \mathrm{~V}$ & $3986^{\mathrm{CTR}}$ & $4.7^{\mathrm{ST}}$ & $0.00^{\mathrm{ST}}$ & 0.62 & 0.84 & 0.22 \\
\hline IRL080 & HD 088639 & 101349.70 & +270808.95 & G3IIIb & $5431^{\mathrm{FSF}}$ & $2.85^{\mathrm{FSF}}$ & $-0.41^{\mathrm{FSF}}$ & 0.44 & 0.50 & 0.06 \\
\hline IRL015 & HD 089025 & 101641.41 & +232502.32 & F0IIIa & $7083^{\mathrm{C} 07}$ & $3.2^{\mathrm{C} 07}$ & $-0.03^{\mathrm{C} 07}$ & 0.18 & 0.21 & 0.02 \\
\hline IRL246 & Gl 388 & 101936.27 & +195212.06 & M3V & $3988^{\mathrm{CTR}}$ & $4.74^{\mathrm{ST}}$ & $0.00^{\mathrm{ST}}$ & 0.62 & 0.84 & 0.22 \\
\hline IRL186 & HD 237903 & 103025.31 & +555956.83 & K7V & $4070^{\mathrm{C} 07}$ & $4.7^{\mathrm{C} 07}$ & $-0.18^{\mathrm{C} 07}$ & 0.66 & 0.78 & 0.12 \\
\hline IRL139 & HD 091810 & 103720.55 & +562552.82 & K1IIIb & $4525^{\mathrm{FSF}}$ & $2.79^{\mathrm{ST}}$ & $-0.06^{\mathrm{FSF}}$ & 0.59 & 0.71 & 0.12 \\
\hline IRL004 & HD 092055 & 103733.27 & -132304.35 & $\mathrm{CN} 4.5$ & $3039^{\mathrm{CTR}}$ & $-0.5^{\mathrm{C} 07}$ & $-0.10^{\mathrm{C} 07}$ & 1.09 & 1.54 & 0.45 \\
\hline IRL283 & DENIS-P J104814.7-395606 & 104814.64 & -395606.24 & M9V & $3069^{\mathrm{CTR}}$ & $5.0^{\mathrm{ST}}$ & $0.00^{\mathrm{ST}}$ & 0.61 & 1.05 & 0.44 \\
\hline IRL085 & HD 094481 & 105417.77 & -134528.94 & G4III & $5347^{\mathrm{FSF}}$ & $2.87^{\mathrm{FSF}}$ & $-0.37^{\mathrm{FSF}}$ & 0.45 & 0.52 & 0.07 \\
\hline IRL256 & HD 094705 & 105601.46 & +061107.32 & M5.5III & $3561^{\mathrm{CTR}}$ & $0.2^{\mathrm{C} 07}$ & $-2.50^{\mathrm{C} 07}$ & 0.91 & 1.21 & 0.31 \\
\hline IRL268 & Gl 406 & 105628.86 & +070052.77 & M6V & $3158^{\mathrm{CTR}}$ & $4.84^{\mathrm{ST}}$ & $0.00^{\mathrm{ST}}$ & 0.64 & 1.03 & 0.39 \\
\hline IRL066 & HD 095128 & 105927.97 & +402548.92 & G1V & $5834^{\mathrm{C} 07}$ & $4.34^{\mathrm{C} 07}$ & $0.04^{\mathrm{C} 07}$ & 0.31 & 0.35 & 0.04 \\
\hline IRL231 & HD 095735 & 110320.19 & +3558 11.56 & M2V & $4050^{\mathrm{CTR}}$ & $4.8^{\mathrm{C} 07}$ & $-0.20^{\mathrm{C} 07}$ & 0.57 & 0.77 & 0.20 \\
\hline IRL157 & HD 099998 ext & 113018.89 & -030012.59 & K3III & $4176^{\mathrm{CTR}}$ & $1.67^{\mathrm{C} 07}$ & $-0.39^{\mathrm{C} 07}$ & 0.73 & 0.86 & 0.13 \\
\hline IRL158 & HD 099998 & 113018.89 & -030012.59 & K3III & $3930^{\text {CTR }}$ & $1.67^{\mathrm{C} 07}$ & $-0.39^{\mathrm{C} 07}$ & 0.82 & 0.99 & 0.17 \\
\hline IRL131 & HD 100006 & 113029.03 & +182435.28 & K0III & $4810^{\mathrm{FSF}}$ & $2.93^{\mathrm{ST}}$ & $-0.32^{\mathrm{FSF}}$ & 0.59 & 0.67 & 0.09 \\
\hline IRL115 & HD 101501 & 114103.01 & +341205.88 & G8V & $5401^{\mathrm{C} 07}$ & $4.6^{\mathrm{C} 07}$ & $-0.13^{\mathrm{C} 07}$ & 0.37 & 0.42 & 0.05 \\
\hline IRL192 & 2MASS J11463449+2230527 & 114634.49 & +223052.74 & L3 & $2170^{\text {CTR }}$ & $\geq 5.0^{\mathrm{ST}}$ & $0.00^{\mathrm{ST}}$ & 0.95 & 1.57 & 0.62 \\
\hline IRL044 & HD 102870 & 115041.71 & +014552.99 & F8.5IV & $6109^{\mathrm{C} 07}$ & $4.2^{\mathrm{C} 07}$ & $0.17^{\mathrm{C} 07}$ & 0.28 & 0.32 & 0.03 \\
\hline IRL112 & HD 104979 & 120512.54 & +084358.74 & G8III & $5030^{\mathrm{FSF}}$ & $2.35^{\mathrm{FSF}}$ & $-0.13^{\mathrm{FSF}}$ & 0.54 & 0.62 & 0.08 \\
\hline IRL281 & BR B1219-1336 & 122152.48 & -135310.20 & M9III & $3487^{\mathrm{CTR}}$ & $0.6^{\mathrm{ST}}$ & $0.00^{\mathrm{ST}}$ & 0.76 & 1.28 & 0.52 \\
\hline IRL018 & HD 108519 & 122746.30 & +272521.95 & F0V & $6608^{\mathrm{FSF}}$ & $4.3^{\mathrm{ST}}$ & $0.00^{\mathrm{ST}}$ & 0.16 & 0.20 & 0.04 \\
\hline IRL084 & HD 108477 & 122749.44 & -163754.63 & G4III & $5442^{\mathrm{FSF}}$ & $3.25^{\mathrm{ST}}$ & $-0.05^{\mathrm{FSF}}$ & 0.45 & 0.53 & 0.09 \\
\hline IRL270 & HD 108849 & 123021.01 & +042459.16 & M7III & $3491^{\mathrm{CTR}}$ & $0.84^{\mathrm{ST}}$ & $0.00^{\mathrm{ST}}$ & 0.92 & 1.30 & 0.38 \\
\hline IRL060 & HD 109358 & 123344.54 & +412126.92 & G0V & $5986^{\mathrm{FSF}}$ & $4.11^{\mathrm{FSF}}$ & $-0.13^{\mathrm{FSF}}$ & 0.33 & 0.35 & 0.02 \\
\hline IRL050 & HD 111844 & 125154.38 & +191005.06 & F8IV & $6524^{\mathrm{FSF}}$ & $3.92^{\mathrm{ST}}$ & $-0.74^{\mathrm{SEC}}$ & 0.13 & 0.13 & 0.00 \\
\hline IRL291 & SDSS J125453.90-012247.4 & 125453.93 & -012247.49 & $\mathrm{~T} 2$ & $1449^{\mathrm{CTR}}$ & $\geq 5.0^{\mathrm{ST}}$ & $0.00^{\mathrm{ST}}$ & 0.76 & 0.92 & 0.16 \\
\hline IRL026 & HD 113139 & 130043.69 & +562158.81 & $\mathrm{~F} 2 \mathrm{~V}$ & $6810^{\mathrm{C} 07}$ & $3.87^{\mathrm{C} 07}$ & $0.22^{\mathrm{C} 07}$ & 0.21 & 0.22 & 0.02 \\
\hline IRL191 & Kelu & 130540.19 & -254105.99 & $\mathrm{~L} 2$ & $2125^{\mathrm{CTR}}$ & $\geq 5.0^{\mathrm{ST}}$ & $0.00^{\mathrm{ST}}$ & 0.95 & 1.60 & 0.66 \\
\hline IRL053 & HD 114710 & 131152.39 & +275241.45 & $\mathrm{~F} 9.5 \mathrm{~V}$ & $5975^{\mathrm{C} 07}$ & $4.4^{\mathrm{C} 07}$ & $0.09^{\mathrm{C} 07}$ & 0.22 & 0.21 & -0.01 \\
\hline IRL154 & HD 114960 & 131357.56 & +012723.20 & K3.5IIIb & $4331^{\mathrm{FSF}}$ & $1.97^{\mathrm{FSF}}$ & $-0.13^{\mathrm{FSF}}$ & 0.68 & 0.82 & 0.13 \\
\hline IRL108 & HD 114946 & 131410.89 & -195551.40 & G7IV & $5171^{\mathrm{C} 07}$ & $3.64^{\mathrm{C} 07}$ & $0.13^{\mathrm{C} 07}$ & 0.50 & 0.58 & 0.08 \\
\hline IRL094 & HD 115617 & 131824.31 & -181840.30 & G6.5V & $5531^{\mathrm{C} 07}$ & $4.32^{\mathrm{C} 07}$ & $-0.01^{\mathrm{C} 07}$ & 0.32 & 0.33 & 0.01 \\
\hline IRL287 & $\mathrm{BD}+442267$ & 132118.73 & +435913.67 & S2.5 & $3926^{\mathrm{CTR}}$ & $0.55^{\mathrm{ST}}$ & $0.00^{\mathrm{ST}}$ & 0.84 & 1.07 & 0.22 \\
\hline IRL229 & HD 120052 & 134725.39 & -175135.42 & M2III & $3608^{\mathrm{CTR}}$ & $1.35^{\mathrm{ST}}$ & $0.00^{\mathrm{ST}}$ & 0.91 & 1.16 & 0.25 \\
\hline IRL176 & HD 120477 & 134928.64 & +154752.46 & K5.5III & $3913^{\mathrm{CTR}}$ & $1.32^{\mathrm{SEC}}$ & $-0.30^{\mathrm{SEC}}$ & 0.81 & 1.00 & 0.19 \\
\hline IRL114 & HD 122563 & 140231.84 & +094109.94 & G8III & $4566^{\mathrm{C} 07}$ & $1.12^{\mathrm{C} 07}$ & $-2.63^{\mathrm{C} 07}$ & 0.58 & 0.68 & 0.10 \\
\hline IRL206 & IRAS $14086-0730$ & 141118.03 & -074447.30 & M10III & $3601^{\mathrm{CTR}}$ & $0.5^{\mathrm{ST}}$ & $0.00^{\mathrm{ST}}$ & 2.15 & 4.12 & 1.98 \\
\hline IRL135 & HD 124897 shape ext & 141539.67 & +191056.67 & K1.5III & $4361^{\mathrm{C} 07}$ & $1.93^{\mathrm{C} 07}$ & $-0.53^{\mathrm{C} 07}$ & 0.68 & 0.77 & 0.09 \\
\hline IRL136 & HD 124897 shape & 141539.67 & +191056.67 & K1.5III & $4361^{\mathrm{C} 07}$ & $1.93^{\mathrm{C} 07}$ & $-0.53^{\mathrm{C} 07}$ & 0.71 & 0.82 & 0.11 \\
\hline IRL134 & HD 124897 lines & 141539.67 & +191056.67 & K1.5III & $4361^{\mathrm{C} 07}$ & $1.93^{\mathrm{C} 07}$ & $-0.53^{\mathrm{C} 07}$ & 0.71 & 0.82 & 0.11 \\
\hline IRL133 & HD 124897 lines ext & 141539.67 & +191056.67 & K1.5III & $4361^{\mathrm{C} 07}$ & $1.93^{\mathrm{C} 07}$ & $-0.53^{\mathrm{C} 07}$ & 0.68 & 0.77 & 0.09 \\
\hline IRL042 & HD 124850 & 141600.86 & -060001.96 & F7III & $6116^{\mathrm{C} 07}$ & $3.83^{\mathrm{C} 07}$ & $-0.11^{\mathrm{C} 07}$ & 0.28 & 0.30 & 0.02 \\
\hline IRL043 & HD 126660 & 142511.79 & +515102.67 & F7V & $6202^{\mathrm{C} 07}$ & $3.84^{\mathrm{C} 07}$ & $-0.27^{\mathrm{C} 07}$ & 0.24 & 0.30 & 0.05 \\
\hline IRL074 & HD 126868 & 142812.13 & -021340.65 & G2IV & $5731^{\mathrm{FSF}}$ & $3.35^{\mathrm{FSF}}$ & $-0.15^{\mathrm{FSF}}$ & 0.36 & 0.42 & 0.06 \\
\hline IRL285 & LH S2924 & 142843.23 & +331039.14 & M9V & $2772^{\mathrm{CTR}}$ & $5.0^{\mathrm{ST}}$ & $0.00^{\mathrm{ST}}$ & 0.76 & 1.26 & 0.50 \\
\hline IRL274 & IRAS 14303 & 143259.89 & -105603.60 & M8III & $3306^{\mathrm{CTR}}$ & $0.7^{\mathrm{ST}}$ & $0.00^{\mathrm{ST}}$ & 0.79 & 1.38 & 0.59 \\
\hline IRL190 & 2MASS J14392836+1929149 & 143928.36 & +192914.98 & $\mathrm{~L} 1$ & $2528^{\mathrm{CTR}}$ & $\geq 5.0^{\mathrm{ST}}$ & $0.00^{\mathrm{ST}}$ & 0.75 & 1.25 & 0.50 \\
\hline IRL275 & IRAS $14436-0703$ & 144618.41 & -071549.80 & M8III & $3465^{\mathrm{CTR}}$ & $0.7^{\mathrm{ST}}$ & $0.00^{\mathrm{ST}}$ & 0.76 & 1.29 & 0.53 \\
\hline
\end{tabular}


Table A.1. continued.

\begin{tabular}{|c|c|c|c|c|c|c|c|c|c|c|}
\hline ID & Star & RA & Dec & Class & $T_{\text {eff }}(\mathrm{K})$ & $\log g$ & {$\left[Z / Z_{\odot}\right]$} & $(J-H)$ & $(J-K)$ & $(H-K)$ \\
\hline IRL149 & HD 132935 & 150204.23 & -082040.99 & K2III & $4547^{\mathrm{FSF}}$ & $2.17^{\mathrm{FSF}}$ & $-0.11^{\mathrm{FSF}}$ & 0.73 & 0.87 & 0.15 \\
\hline IRL148 & HD 132935 ext & 150204.23 & -082040.99 & K2III & $4502^{\mathrm{FSF}}$ & $2.68^{\mathrm{ST}}$ & $-0.07^{\mathrm{FSF}}$ & 0.66 & 0.78 & 0.11 \\
\hline IRL193 & 2MASS J15065441+1321060 & 150654.41 & +132106.08 & L3 & $2064^{\mathrm{CTR}}$ & $\geq 5.0^{\mathrm{ST}}$ & $0.00^{\mathrm{ST}}$ & 0.97 & 1.65 & 0.67 \\
\hline IRL196 & 2MASS J15074769-1627386 & 150747.69 & -162738.62 & L5 & $2234^{\mathrm{CTR}}$ & $\geq 5.0^{\mathrm{ST}}$ & $0.00^{\mathrm{ST}}$ & 0.96 & 1.53 & 0.57 \\
\hline IRL282 & IRAS $15060+0947$ & 150825.77 & +093618.20 & M9III & $2203^{\mathrm{CTR}}$ & $0.6^{\mathrm{ST}}$ & $0.00^{\mathrm{ST}}$ & 1.30 & 2.45 & 1.15 \\
\hline IRL012 & HD 135153 & 151437.31 & -313108.85 & FOIb & $7073^{\mathrm{FSF}}$ & $2.07^{\mathrm{FSF}}$ & $-0.06^{\mathrm{SEC}}$ & 0.21 & 0.29 & 0.08 \\
\hline IRL011 & HD 135153 ext & 151437.31 & -313108.85 & FOIb & $7037^{\mathrm{FSF}}$ & $2.03^{\mathrm{FSF}}$ & $-0.06^{\mathrm{SEC}}$ & 0.15 & 0.19 & 0.04 \\
\hline IRL198 & 2MASS J15150083+4847416 & 151500.83 & +484741.69 & L6 & $2014^{\mathrm{CTR}}$ & $\geq 5.0^{\mathrm{ST}}$ & $0.00^{\mathrm{ST}}$ & 1.04 & 1.68 & 0.64 \\
\hline IRL113 & HD 135722 & 151530.16 & +331853.39 & G8III & $4847^{\mathrm{C} 07}$ & $2.56^{\mathrm{C} 07}$ & $-0.44^{\mathrm{C} 07}$ & 0.54 & 0.65 & 0.11 \\
\hline IRL222 & Gl 581 & 151927.50 & -074319.44 & $\mathrm{M} 2.5 \mathrm{~V}$ & $3966^{\mathrm{CTR}}$ & $4.7^{\mathrm{ST}}$ & $0.00^{\mathrm{ST}}$ & 0.59 & 0.84 & 0.26 \\
\hline IRL150 & HD 137759 & 152455.77 & +58 5757.83 & $\mathrm{~K} 2 \mathrm{III}$ & $4498^{\mathrm{C} 07}$ & $2.38^{\mathrm{C} 07}$ & $0.05^{\mathrm{C} 07}$ & 0.63 & 0.75 & 0.12 \\
\hline IRL263 & HD 142143 & 155046.62 & +482858.85 & M6.5III & $3443^{\mathrm{CTR}}$ & $0.88^{\mathrm{ST}}$ & $0.00^{\mathrm{ST}}$ & 0.96 & 1.36 & 0.40 \\
\hline IRL142 & HD 142091 & 155113.93 & +353926.56 & K1IVa & $4796^{\mathrm{C} 07}$ & $3.22^{\mathrm{C} 07}$ & $0.00^{\mathrm{C} 07}$ & 0.51 & 0.58 & 0.07 \\
\hline IRL132 & HD 145675 & 161024.31 & +434903.52 & K0V & $5264^{\mathrm{C} 07}$ & $4.66^{\mathrm{C} 07}$ & $0.34^{\mathrm{C} 07}$ & 0.39 & 0.43 & 0.05 \\
\hline IRL022 & $\mathrm{BD}+382803$ & 163557.28 & +375802.10 & F2Ib & $6588^{\mathrm{FSF}}$ & $1.87^{\mathrm{FSF}}$ & $0.00^{\mathrm{ST}}$ & 0.34 & 0.41 & 0.08 \\
\hline IRL273 & Gl 644 & 165528.75 & -082010.78 & M7V & $3278^{\mathrm{CTR}}$ & $4.9^{\mathrm{ST}}$ & $0.00^{\mathrm{ST}}$ & 0.59 & 1.00 & 0.41 \\
\hline IRL258 & HD 156014 & 171438.87 & +142325.01 & M5Ib & $4135^{\mathrm{CTR}}$ & $0.76^{\mathrm{C} 07}$ & $-2.50^{\mathrm{C} 07}$ & 0.68 & 0.76 & 0.08 \\
\hline IRL038 & HD 160365 & 173857.85 & +131945.34 & F6III & $6070^{\mathrm{C} 07}$ & $3.0^{\mathrm{C} 07}$ & $-0.26^{\mathrm{C} 07}$ & 0.20 & 0.16 & -0.04 \\
\hline IRL095 & HD 161664 ext & 174745.60 & -222840.05 & G6Ib & $4739^{\mathrm{FSF}}$ & $1.64^{\mathrm{FSF}}$ & $-0.07^{\mathrm{FSF}}$ & 0.50 & 0.58 & 0.08 \\
\hline IRL096 & HD 161664 & 174745.60 & -222840.05 & G6Ib & $4804^{\mathrm{FSF}}$ & $1.63^{\mathrm{FSF}}$ & $-0.09^{\mathrm{FSF}}$ & 0.63 & 0.80 & 0.16 \\
\hline IRL187 & HD 164136 & 175830.14 & +301121.38 & F2IV & $6799^{\mathrm{C} 07}$ & $2.63^{\mathrm{C} 07}$ & $-0.30^{\mathrm{C} 07}$ & 0.24 & 0.26 & 0.02 \\
\hline IRL121 & HD 164349 ext & 180003.41 & +164503.30 & $\mathrm{~K} 0.5 \mathrm{IIb}$ & $4446^{\mathrm{C} 07}$ & $1.5^{\mathrm{C} 07}$ & $0.39^{\mathrm{C} 07}$ & 0.49 & 0.52 & 0.03 \\
\hline IRL122 & HD 164349 & 180003.41 & +164503.30 & $\mathrm{~K} 0.5 \mathrm{IIb}$ & $4446^{\mathrm{C} 07}$ & $1.5^{\mathrm{C} 07}$ & $0.39^{\mathrm{C} 07}$ & 0.53 & 0.59 & 0.06 \\
\hline IRL143 & HD 165438 & 180615.19 & -044504.51 & K1IV & $4862^{\mathrm{C} 07}$ & $3.4^{\mathrm{C} 07}$ & $0.02^{\mathrm{C} 07}$ & 0.51 & 0.59 & 0.08 \\
\hline IRL093 & HD 165185 & 180623.71 & -360111.23 & G5V & $5974^{\mathrm{FSF}}$ & $3.85^{\mathrm{FSF}}$ & $-0.15^{\mathrm{FSF}}$ & 0.29 & 0.34 & 0.05 \\
\hline IRL057 & HD 165908 & 180701.53 & +303343.68 & F9V & $5928^{\mathrm{C} 07}$ & $4.24^{\mathrm{C} 07}$ & $-0.53^{\mathrm{C} 07}$ & 0.26 & 0.31 & 0.05 \\
\hline IRL125 & HD 165782 ext & 180826.51 & -183307.92 & K0Ia & $5211^{\mathrm{FSF}}$ & $0.25^{\mathrm{ST}}$ & $0.20^{\mathrm{FSF}}$ & 0.34 & 0.44 & 0.10 \\
\hline IRL126 & HD 165782 & 180826.51 & -183307.92 & K0Ia & $5600^{\mathrm{FSF}}$ & $0.25^{\mathrm{ST}}$ & $0.21^{\mathrm{FSF}}$ & 0.63 & 0.90 & 0.27 \\
\hline IRL119 & HD 170820 & 183213.11 & -190726.28 & G9II & $4604^{\mathrm{C} 07}$ & $1.62^{\mathrm{C} 07}$ & $0.17^{\mathrm{C} 07}$ & 0.69 & 0.89 & 0.20 \\
\hline IRL118 & HD 170820 ext & 183213.11 & -190726.28 & G9II & $4604^{\mathrm{C} 07}$ & $1.62^{\mathrm{C} 07}$ & $0.17^{\mathrm{C} 07}$ & 0.53 & 0.63 & 0.10 \\
\hline IRL019 & HD 173638 ext & 184643.32 & -100730.17 & F1II & $7005^{\mathrm{FSF}}$ & $2.1^{\mathrm{FSF}}$ & $-0.68^{\mathrm{FSF}}$ & 0.14 & 0.14 & 0.00 \\
\hline IRL020 & HD 173638 & 184643.32 & -100730.17 & F1II & $7146^{\mathrm{FSF}}$ & $2.2^{\mathrm{FSF}}$ & $-0.56^{\mathrm{FSF}}$ & 0.25 & 0.32 & 0.07 \\
\hline IRL259 & HD 175865 & 185520.10 & +435645.93 & M5III & $3690^{\mathrm{CTR}}$ & $0.5^{\mathrm{C} 07}$ & $0.14^{\mathrm{C} 07}$ & 0.87 & 1.08 & 0.21 \\
\hline IRL056 & HD 176051 & 185701.60 & +325404.57 & F9V & $5895^{\mathrm{FSF}}$ & $3.91^{\mathrm{FSF}}$ & $-0.21^{\mathrm{FSF}}$ & 0.36 & 0.40 & 0.05 \\
\hline IRL078 & HD 176123 ext & 185926.77 & -183359.16 & G3II & $5449^{\mathrm{FSF}}$ & $2.8^{\mathrm{FSF}}$ & $-0.23^{\mathrm{FSF}}$ & 0.42 & 0.48 & 0.06 \\
\hline IRL079 & HD 176123 & 185926.77 & -183359.16 & G3II & $5302^{\mathrm{FSF}}$ & $2.61^{\mathrm{FSF}}$ & $-0.30^{\mathrm{FSF}}$ & 0.46 & 0.54 & 0.08 \\
\hline IRL161 & HD 178208 ext & 190509.83 & +495523.39 & K3III & $4882^{\mathrm{FSF}}$ & $2.53^{\mathrm{FSF}}$ & $-0.26^{\mathrm{FSF}}$ & 0.62 & 0.72 & 0.10 \\
\hline IRL162 & HD 178208 & 190509.83 & +495523.39 & K3III & $4884^{\mathrm{FSF}}$ & $2.48^{\mathrm{FSF}}$ & $-0.23^{\mathrm{FSF}}$ & 0.66 & 0.78 & 0.13 \\
\hline IRL129 & HD 179870 ext & 191353.58 & +090159.59 & KOII & $4776^{\mathrm{FSF}}$ & $1.53^{\mathrm{FSF}}$ & $0.04^{\mathrm{FSF}}$ & 0.44 & 0.44 & 0.00 \\
\hline IRL130 & HD 179870 & 191353.58 & +090159.59 & KOII & $4928^{\mathrm{FSF}}$ & $1.57^{\mathrm{FSF}}$ & $0.06^{\mathrm{FSF}}$ & 0.49 & 0.53 & 0.04 \\
\hline IRL086 & HD 179821 ext & 191358.60 & +000731.92 & G4Ia & $5708^{\mathrm{CTR}}$ & $1.08^{\mathrm{FSF}}$ & $0.00^{\mathrm{ST}}$ & 0.25 & 0.38 & 0.13 \\
\hline IRL087 & HD 179821 & 191358.60 & +000731.92 & G4Ia & $4645^{\mathrm{CTR}}$ & $0.95^{\mathrm{FSF}}$ & $0.00^{\mathrm{ST}}$ & 0.43 & 0.67 & 0.24 \\
\hline IRL278 & Gl 752 & 191655.26 & +051008.10 & M8V & $2970^{\mathrm{CTR}}$ & $4.96^{\mathrm{ST}}$ & $0.00^{\mathrm{ST}}$ & 0.64 & 1.09 & 0.45 \\
\hline IRL179 & HD 181596 & 191830.12 & +501339.42 & K5III & $4275^{\mathrm{FSF}}$ & $2.35^{\mathrm{ST}}$ & $-0.41^{\mathrm{FSF}}$ & 0.80 & 0.98 & 0.18 \\
\hline IRL182 & HD 181475 ext & 192048.31 & -043009.01 & K7IIa & $3996^{\mathrm{FSF}}$ & $0.65^{\mathrm{FSF}}$ & $-0.37^{\mathrm{FSF}}$ & 0.78 & 0.96 & 0.19 \\
\hline IRL183 & HD 181475 & 192048.31 & -043009.01 & K7IIa & $4124^{\mathrm{FSF}}$ & $0.83^{\mathrm{FSF}}$ & $-0.21^{\mathrm{FSF}}$ & 0.94 & 1.22 & 0.28 \\
\hline IRL107 & HD 182694 & 192356.50 & +432317.41 & G7III & $5312^{\mathrm{FSF}}$ & $2.63^{\mathrm{FSF}}$ & $-0.19^{\mathrm{FSF}}$ & 0.45 & 0.55 & 0.10 \\
\hline IRL024 & HD 182835 & 192631.09 & +002018.85 & $\mathrm{~F} 2 \mathrm{Ib}$ & $7350^{\mathrm{C} 07}$ & $2.15^{\mathrm{C} 07}$ & $0.09^{\mathrm{C} 07}$ & 0.25 & 0.31 & 0.06 \\
\hline IRL023 & HD 182835 ext & 192631.09 & +002018.85 & F2Ib & $7350^{\mathrm{C} 07}$ & $2.15^{\mathrm{C} 07}$ & $0.09^{\mathrm{C} 07}$ & 0.12 & 0.11 & -0.01 \\
\hline IRL059 & HD 185018 & 193652.45 & +111623.53 & G0Ib & $5550^{\mathrm{C} 07}$ & $1.3^{\mathrm{C} 07}$ & $-0.24^{\mathrm{C} 07}$ & 0.41 & 0.48 & 0.07 \\
\hline IRL058 & HD 185018 ext & 193652.45 & +111623.53 & G0Ib & $5550^{\mathrm{C} 07}$ & $1.3^{\mathrm{C} 07}$ & $-0.24^{\mathrm{C} 07}$ & 0.37 & 0.43 & 0.05 \\
\hline IRL168 & HD 185622 ext & 193925.33 & +163416.03 & $\mathrm{~K} 4 \mathrm{Ib}$ & $3736^{\mathrm{FSF}}$ & $1.05^{\mathrm{ST}}$ & $-0.19^{\mathrm{FSF}}$ & 0.74 & 0.92 & 0.18 \\
\hline IRL169 & HD 185622 & 193925.33 & +163416.03 & $\mathrm{~K} 4 \mathrm{Ib}$ & $3898^{\mathrm{FSF}}$ & $0.63^{\mathrm{FSF}}$ & $-0.14^{\mathrm{FSF}}$ & 0.90 & 1.17 & 0.27 \\
\hline IRL035 & HD 186155 & 194050.18 & +453129.78 & F5II & $6590^{\mathrm{FSF}}$ & $3.34^{\mathrm{FSF}}$ & $0.31^{\mathrm{FSF}}$ & 0.18 & 0.22 & 0.03 \\
\hline IRL156 & HD 187238 & 194811.83 & +224546.35 & K3Iab & $4143^{\mathrm{FSF}}$ & $0.99^{\mathrm{FSF}}$ & $-0.01^{\mathrm{FSF}}$ & 0.81 & 1.02 & 0.21 \\
\hline IRL155 & HD 187238 ext & 194811.83 & +224546.35 & K3Iab & $4228^{\mathrm{FSF}}$ & $0.77^{\mathrm{FSF}}$ & $-0.09^{\mathrm{FSF}}$ & 0.63 & 0.73 & 0.10 \\
\hline IRL214 & HD 339034 ext & 195011.93 & +245524.19 & M1Ia & $3960^{\mathrm{CTR}}$ & $-0.0^{\mathrm{ST}}$ & $0.00^{\mathrm{ST}}$ & 0.64 & 0.87 & 0.22 \\
\hline IRL215 & HD 339034 & 195011.93 & +245524.19 & M1Ia & $3342^{\mathrm{CTR}}$ & $-0.0^{\mathrm{ST}}$ & $0.00^{\mathrm{ST}}$ & 1.05 & 1.51 & 0.46 \\
\hline IRL089 & HD 190113 ext & 200202.85 & +353828.01 & G5Ib & $4968^{\mathrm{FSF}}$ & $1.45^{\mathrm{FSF}}$ & $-0.28^{\mathrm{FSF}}$ & 0.45 & 0.49 & 0.05 \\
\hline
\end{tabular}


S. Meneses-Goytia et al.: Single stellar populations in the near-IR. I.

Table A.1. continued.

\begin{tabular}{|c|c|c|c|c|c|c|c|c|c|c|}
\hline ID & Star & RA & Dec & Class & $T_{\text {eff }}(\mathrm{K})$ & $\log g$ & {$\left[Z / Z_{\odot}\right]$} & $(J-H)$ & $(J-K)$ & $(H-K)$ \\
\hline IRL090 & HD 190113 & 200202.85 & +353828.01 & G5Ib & $4996^{\mathrm{FSF}}$ & $1.59^{\mathrm{FSF}}$ & $-0.31^{\mathrm{FSF}}$ & 0.60 & 0.73 & 0.14 \\
\hline IRL104 & HD 333385 & 200227.37 & +300425.46 & G7Ia & $5375^{\mathrm{FSF}}$ & $0.36^{\mathrm{ST}}$ & $0.14^{\mathrm{FSF}}$ & 0.61 & 0.90 & 0.29 \\
\hline IRL103 & HD 333385 ext & 200227.37 & +300425.46 & G7Ia & $5475^{\mathrm{FSF}}$ & $1.05^{\mathrm{FSF}}$ & $0.21^{\mathrm{FSF}}$ & 0.29 & 0.38 & 0.10 \\
\hline IRL046 & HD 190323 & 200349.61 & +145858.74 & F8Ia & $5740^{\mathrm{CTR}}$ & $0.81^{\mathrm{ST}}$ & $0.07^{\mathrm{FSF}}$ & 0.32 & 0.38 & 0.06 \\
\hline IRL045 & HD 190323 ext & 200349.61 & +145858.74 & F8Ia & $6906^{\mathrm{FSF}}$ & $0.81^{\mathrm{ST}}$ & $0.09^{\mathrm{FSF}}$ & 0.22 & 0.22 & 0.00 \\
\hline IRL077 & HD 192713 & 201530.23 & +233032.05 & G3Ib & $4864^{\mathrm{C} 07}$ & $0.1^{\mathrm{C} 07}$ & $-0.43^{\mathrm{C} 07}$ & 0.48 & 0.57 & 0.09 \\
\hline IRL076 & HD 192713 ext & 201530.23 & +233032.05 & G3Ib & $4864^{\mathrm{C} 07}$ & $0.1^{\mathrm{C} 07}$ & $-0.43^{\mathrm{C} 07}$ & 0.44 & 0.50 & 0.07 \\
\hline IRL184 & HD 194193 & 202245.29 & +410133.64 & K7III & $4025^{\mathrm{FSF}}$ & $1.42^{\mathrm{FSF}}$ & $-0.14^{\mathrm{FSF}}$ & 0.88 & 1.09 & 0.20 \\
\hline IRL091 & HD 193896 & 202300.79 & -093916.95 & G5IIIa & $5332^{\mathrm{FSF}}$ & $3.2^{\mathrm{ST}}$ & $-0.49^{\mathrm{FSF}}$ & 0.50 & 0.61 & 0.11 \\
\hline IRL244 & RW Cyg ext & 202850.59 & +395854.42 & M3Iab & $3844^{\mathrm{CTR}}$ & $0.16^{\mathrm{ST}}$ & $0.00^{\mathrm{ST}}$ & 0.74 & 0.95 & 0.21 \\
\hline IRL245 & RW Cyg & 202850.59 & +395854.42 & M3Iab & $3341^{\mathrm{CTR}}$ & $0.16^{\mathrm{ST}}$ & $0.00^{\mathrm{ST}}$ & 1.09 & 1.51 & 0.42 \\
\hline IRL267 & HD 196610 & 203754.72 & +181606.89 & M6III & $3643^{\mathrm{CTR}}$ & $0.91^{\mathrm{ST}}$ & $0.00^{\mathrm{ST}}$ & 0.86 & 1.12 & 0.26 \\
\hline IRL230 & G1 806 & 204504.09 & +442956.66 & $\mathrm{M} 2 \mathrm{~V}$ & $3660^{\mathrm{FSF}}$ & $4.65^{\mathrm{FSF}}$ & $-0.23^{\mathrm{FSF}}$ & 0.62 & 0.78 & 0.17 \\
\hline IRL170 & HD 201065 ext & 210535.78 & +465747.76 & $\mathrm{~K} 4 \mathrm{Ib}$ & $4068^{\mathrm{FSF}}$ & $1.16^{\mathrm{FSF}}$ & $-0.50^{\mathrm{FSF}}$ & 0.73 & 0.88 & 0.15 \\
\hline IRL171 & HD 201065 & 210535.78 & +465747.76 & $\mathrm{~K} 4 \mathrm{Ib}$ & $4226^{\mathrm{FSF}}$ & $0.87^{\mathrm{FSF}}$ & $-0.47^{\mathrm{FSF}}$ & 0.83 & 1.04 & 0.20 \\
\hline IRL041 & HD 201078 & 210630.24 & +311104.76 & F7II & $6157^{\mathrm{C} 07}$ & $1.65^{\mathrm{C} 07}$ & $0.13^{\mathrm{C} 07}$ & 0.26 & 0.33 & 0.07 \\
\hline IRL185 & HD 201092 & 210655.26 & +384431.40 & $\mathrm{~K} 7 \mathrm{~V}$ & $4348^{\mathrm{CTR}}$ & $4.37^{\mathrm{SEC}}$ & $-0.72^{\mathrm{SEC}}$ & 0.59 & 0.73 & 0.14 \\
\hline IRL098 & HD 202314 & 211410.28 & +295403.45 & G6Ib & $4864^{\mathrm{C} 07}$ & $1.3^{\mathrm{C} 07}$ & $-0.05^{\mathrm{C} 07}$ & 0.51 & 0.66 & 0.14 \\
\hline IRL097 & HD 202314 ext & 211410.28 & +295403.45 & G6Ib & $4864^{\mathrm{C} 07}$ & $1.3^{\mathrm{C} 07}$ & $-0.05^{\mathrm{C} 07}$ & 0.48 & 0.60 & 0.12 \\
\hline IRL247 & HD 204585 & 212859.77 & +221045.96 & M4.5IIIa & $3700^{\mathrm{CTR}}$ & $1.11^{\mathrm{ST}}$ & $0.00^{\mathrm{ST}}$ & 0.86 & 1.07 & 0.21 \\
\hline IRL216 & HD 204724 & 212956.89 & +233819.81 & M1III & $3765^{\mathrm{CTR}}$ & $1.5^{\mathrm{ST}}$ & $0.00^{\mathrm{ST}}$ & 0.78 & 1.01 & 0.23 \\
\hline IRL276 & IRAS 21284-0747 & 213106.51 & -073420.50 & M8III & $3130^{\mathrm{CTR}}$ & $0.7^{\mathrm{ST}}$ & $0.00^{\mathrm{ST}}$ & 0.87 & 1.48 & 0.61 \\
\hline IRL224 & HD 206936 & 214330.46 & +584648.16 & M2Ia & $3540^{\mathrm{CTR}}$ & $0.0^{\mathrm{ST}}$ & $0.00^{\mathrm{ST}}$ & 0.86 & 1.24 & 0.38 \\
\hline IRL223 & HD 206936 ext & 214330.46 & +584648.16 & M2Ia & $3879^{\mathrm{CTR}}$ & $0.0^{\mathrm{ST}}$ & $0.00^{\mathrm{ST}}$ & 0.66 & 0.92 & 0.26 \\
\hline IRL271 & HD 207076 & 214631.84 & -021245.93 & M7III & $2750^{\mathrm{C} 07}$ & $-0.5^{\mathrm{C} 07}$ & $-2.50^{\mathrm{C} 07}$ & 0.78 & 1.03 & 0.25 \\
\hline IRL172 & HD 207991 ext & 215155.38 & +482613.59 & K4III & $4355^{\mathrm{FSF}}$ & $1.98^{\mathrm{FSF}}$ & $-0.21^{\mathrm{FSF}}$ & 0.79 & 0.94 & 0.15 \\
\hline IRL173 & HD 207991 & 215155.38 & +482613.59 & K4III & $4192^{\mathrm{FSF}}$ & $2.48^{\mathrm{ST}}$ & $-0.32^{\mathrm{FSF}}$ & 0.84 & 1.02 & 0.18 \\
\hline IRL111 & HD 208606 & 215520.59 & +613230.52 & G8Ib & $4718^{\mathrm{FSF}}$ & $0.66^{\mathrm{FSF}}$ & $-0.16^{\mathrm{FSF}}$ & 0.67 & 0.86 & 0.19 \\
\hline IRL110 & HD 208606 ext & 215520.59 & +613230.52 & G8Ib & $4709^{\mathrm{FSF}}$ & $1.26^{\mathrm{FSF}}$ & $0.10^{\mathrm{FSF}}$ & 0.54 & 0.64 & 0.10 \\
\hline IRL203 & HD 209290 & 220210.27 & +012400.82 & $\mathrm{M} 0.5 \mathrm{~V}$ & $3810^{\mathrm{CTR}}$ & $4.65^{\mathrm{ST}}$ & $0.00^{\mathrm{ST}}$ & 0.68 & 0.88 & 0.20 \\
\hline IRL152 & HD 212466 & 222307.01 & +55 5747.62 & K2Ia & $3749^{\mathrm{CTR}}$ & $0.2^{\mathrm{ST}}$ & $0.18^{\mathrm{FSF}}$ & 0.73 & 1.10 & 0.37 \\
\hline IRL151 & HD $212466 \mathrm{ext}$ & 222307.01 & +555747.62 & K2Ia & $5018^{\mathrm{FSF}}$ & $0.2^{\mathrm{ST}}$ & $0.02^{\mathrm{FSF}}$ & 0.42 & 0.61 & 0.19 \\
\hline IRL195 & 2MASS J22244381-0158521 & 222443.81 & -015852.14 & L4.5 & $1267^{\mathrm{CTR}}$ & $\geq 5.0^{\mathrm{ST}}$ & $0.00^{\mathrm{ST}}$ & 1.26 & 2.06 & 0.81 \\
\hline IRL033 & HD 213306 & 222910.26 & +582454.71 & F5Ib & $6052^{\mathrm{FSF}}$ & $2.29^{\mathrm{FSF}}$ & $-0.16^{\mathrm{FSF}}$ & 0.40 & 0.47 & 0.07 \\
\hline IRL032 & HD 213306 ext & 222910.26 & +582454.71 & F5Ib & $6052^{\mathrm{FSF}}$ & $2.27^{\mathrm{FSF}}$ & $-0.14^{\mathrm{FSF}}$ & 0.34 & 0.37 & 0.03 \\
\hline IRL021 & HD 213135 & 222946.02 & -270626.22 & F1V & $6404^{\mathrm{FSF}}$ & $4.3^{\mathrm{ST}}$ & $-0.54^{\mathrm{FSF}}$ & 0.20 & 0.21 & 0.01 \\
\hline IRL204 & HD 213893 & 223435.93 & +003542.63 & M0IIIb & $4044^{\mathrm{C} 07}$ & $1.6^{\mathrm{C} 07}$ & $-0.08^{\mathrm{C} 07}$ & 0.83 & 1.01 & 0.18 \\
\hline IRL261 & Gl 866 ext & 223833.72 & -151757.33 & M5V & $3728^{\mathrm{CTR}}$ & $4.8^{\mathrm{ST}}$ & $0.00^{\mathrm{ST}}$ & 0.59 & 0.90 & 0.31 \\
\hline IRL262 & Gl 866 & 223833.72 & -151757.33 & M5V & $3369^{\mathrm{CTR}}$ & $4.8^{\mathrm{ST}}$ & $0.00^{\mathrm{ST}}$ & 0.64 & 0.98 & 0.34 \\
\hline IRL250 & HD 214665 & 223837.92 & +564744.28 & M4III & $3452^{\mathrm{CTR}}$ & $1.07^{\mathrm{ST}}$ & $0.00^{\mathrm{ST}}$ & 1.04 & 1.35 & 0.31 \\
\hline IRL088 & HD 214850 & 224052.68 & +143256.97 & G4V & $5451^{\mathrm{FSF}}$ & $4.48^{\mathrm{ST}}$ & $-0.34^{\mathrm{FSF}}$ & 0.44 & 0.53 & 0.09 \\
\hline IRL040 & HD 215648 & 224641.58 & +121022.38 & F6V & $6167^{\mathrm{C} 07}$ & $4.04^{\mathrm{C} 07}$ & $-0.32^{\mathrm{C} 07}$ & 0.26 & 0.29 & 0.03 \\
\hline IRL065 & HD 216219 & 225052.15 & +180007.56 & G1II & $5727^{\mathrm{C} 07}$ & $3.36^{\mathrm{C} 07}$ & $-0.39^{\mathrm{C} 07}$ & 0.30 & 0.35 & 0.05 \\
\hline IRL272 & MY Cep & 225431.71 & +604938.89 & M7I & $2595^{\mathrm{CTR}}$ & $-0.2^{\mathrm{ST}}$ & $0.00^{\mathrm{ST}}$ & 1.47 & 2.24 & 0.77 \\
\hline IRL178 & HD 216946 & 225625.99 & +494400.75 & $\mathrm{~K} 5 \mathrm{Ib}$ & $3839^{\mathrm{FSF}}$ & $0.49^{\mathrm{FSF}}$ & $-0.10^{\mathrm{SEC}}$ & 0.95 & 1.24 & 0.29 \\
\hline IRL177 & HD 216946 ext & 225625.99 & +494400.75 & $\mathrm{~K} 5 \mathrm{Ib}$ & $3817^{\mathrm{FSF}}$ & $0.68^{\mathrm{FSF}}$ & $-0.10^{\mathrm{SEC}}$ & 0.90 & 1.16 & 0.26 \\
\hline IRL036 & HD 218804 & 231027.20 & +433239.15 & F5V & $6261^{\mathrm{C} 07}$ & $4.05^{\mathrm{C} 07}$ & $-0.23^{\mathrm{C} 07}$ & 0.27 & 0.32 & 0.05 \\
\hline IRL167 & HD 219134 & 231316.97 & +571006.08 & $\mathrm{K} 3 \mathrm{~V}$ & $4717^{\mathrm{C} 07}$ & $4.5^{\mathrm{C} 07}$ & $0.05^{\mathrm{C} 07}$ & 0.56 & 0.67 & 0.10 \\
\hline IRL073 & HD 219477 & 231546.29 & +281452.43 & G2II & $5989^{\mathrm{FSF}}$ & $2.87^{\mathrm{FSF}}$ & $-0.23^{\mathrm{FSF}}$ & 0.36 & 0.41 & 0.05 \\
\hline IRL051 & HD 219623 & 231642.30 & +531248.51 & F8V & $6155^{\mathrm{C} 07}$ & $4.17^{\mathrm{C} 07}$ & $-0.04^{\mathrm{C} 07}$ & 0.29 & 0.34 & 0.05 \\
\hline IRL220 & HD 219734 & 231744.64 & +490055.08 & M2.5III & $3658^{\mathrm{CTR}}$ & $0.9^{\mathrm{C} 07}$ & $0.27^{\mathrm{C} 07}$ & 0.89 & 1.11 & 0.22 \\
\hline IRL049 & HD 220657 & 232522.78 & +232414.76 & F8III & $6380^{\mathrm{FSF}}$ & $2.96^{\mathrm{FSF}}$ & $-0.66^{\mathrm{FSF}}$ & 0.36 & 0.47 & 0.10 \\
\hline IRL164 & HD 221246 & 233007.41 & +490759.31 & K3III & $4359^{\mathrm{FSF}}$ & $2.58^{\mathrm{ST}}$ & $-0.35^{\mathrm{FSF}}$ & 0.73 & 0.85 & 0.13 \\
\hline IRL163 & HD 221246 ext & 233007.41 & +490759.31 & K3III & $4308^{\mathrm{FSF}}$ & $1.98^{\mathrm{FSF}}$ & $-0.19^{\mathrm{FSF}}$ & 0.67 & 0.76 & 0.09 \\
\hline IRL120 & HD 222093 & 233739.55 & -130336.86 & G9III & $4750^{\mathrm{FSF}}$ & $2.01^{\mathrm{FSF}}$ & $0.03^{\mathrm{FSF}}$ & 0.56 & 0.64 & 0.09 \\
\hline IRL269 & BRI B2339-0447 & 234202.75 & -043104.88 & M7III & $3425^{\mathrm{CTR}}$ & $0.84^{\mathrm{ST}}$ & $0.00^{\mathrm{ST}}$ & 0.93 & 1.39 & 0.46 \\
\hline
\end{tabular}


Table A.2. Additional stars from the MILES and CaT stellar libraries used as templates in the determination of the IRTF stellar temperatures, gravities and metallicities.

\begin{tabular}{cccr}
\hline \hline Star & $T_{\text {eff }}(\mathrm{K})$ & $\log g$ & {$\left[Z / Z_{\odot}\right]$} \\
\hline BD442051 & 3696 & 5.00 & -1.50 \\
HD058521 & 3238 & 0.00 & -0.19 \\
HD069267 & 4043 & 1.51 & -0.12 \\
HD073394 & 4500 & 1.10 & -1.38 \\
HD073593 & 4717 & 2.25 & -0.12 \\
HD076813 & 6072 & 4.20 & -0.82 \\
HD078712 & 3202 & 0.00 & -0.11 \\
HD079452 & 4829 & 2.35 & -0.84 \\
HD081192 & 4705 & 2.50 & -0.62 \\
HD083425 & 4120 & 2.00 & -0.35 \\
HD083618 & 4231 & 1.74 & -0.08 \\
HD083632 & 4214 & 1.00 & -1.39 \\
HD087737 & 9625 & 1.98 & -0.04 \\
HD095735 & 3551 & 4.90 & -0.20 \\
HD096360 & 3550 & 0.50 & -0.58 \\
HD099998 & 3863 & 1.79 & -0.16 \\
HD103095 & 5025 & 4.56 & -1.36 \\
HD107213 & 6298 & 4.01 & 0.36 \\
HD111631 & 3785 & 4.75 & 0.10 \\
HD114038 & 4530 & 2.71 & -0.04 \\
HD114961 & 3012 & 0.00 & -0.81 \\
HD119228 & 3600 & 1.60 & 0.30 \\
HD119667 & 3700 & 1.00 & -0.35 \\
HD120933 & 3820 & 1.52 & 0.50 \\
HD121299 & 4710 & 2.64 & -0.03 \\
HD123657 & 3450 & 0.85 & 0.00 \\
HD126327 & 2819 & 0.00 & -0.58 \\
HD130705 & 4336 & 2.10 & 0.41 \\
HD131430 & 4190 & 2.18 & 0.04 \\
HD134063 & 4885 & 2.34 & -0.69 \\
HD136726 & 4120 & 2.03 & 0.07 \\
HD137704 & 4095 & 1.97 & -0.27 \\
HD138481 & 3890 & 1.64 & 0.20 \\
HD145675 & 5264 & 4.66 & 0.34 \\
HD147923 & 3600 & 0.80 & -0.19 \\
HD148783 & 3279 & 0.20 & -0.06 \\
HD149661 & 5168 & 4.63 & 0.04 \\
HD154733 & 4279 & 2.10 & 0.00 \\
HD164058 & 3930 & 1.26 & -0.05 \\
HD167768 & 5235 & 1.61 & -0.68 \\
HD168720 & 3810 & 1.10 & 0.00 \\
HD184499 & 5738 & 4.02 & -0.66 \\
HD184786 & 3467 & 0.60 & -0.04 \\
HD185144 & 5260 & 4.55 & -0.24 \\
HD187216 & 3500 & 0.40 & -2.48 \\
HD191277 & 4459 & 2.71 & 0.30 \\
\hline HD 32078 & 4000 & 0.30 & -0.24 \\
& & & -1.73 \\
\hline
\end{tabular}

Notes. The stellar parameters were determined by Cenarro et al. (2007).

\section{References}

Allard, F., Homeier, D., Freytag, B., \& Sharp, C. M. 2012, in EAS Pub. Ser., 57, 3

Alonso, A., Arribas, S., \& Martinez-Roger, C. 1996, A\&AS, 117, 227
Alonso, A., Arribas, S., \& Martinez-Roger, C. 1999, A\&AS, 140, 261

Aringer, B., Girardi, L., Nowotny, W., Marigo, P., \& Lederer, M. T. 2009, A\&A, 503, 913

Bessell, M. S., Castelli, F., \& Plez, B. 1998, A\&A, 333, 231

Burstein, D., Faber, S. M., Gaskell, C. M., \& Krumm, N. 1984, ApJ, 287, 586 Cappellari, M., \& Emsellem, E. 2004, PASP, 116, 138

Cayrel de Strobel, G., Soubiran, C., Friel, E. D., Ralite, N., \& Francois, P. 1997, A\&AS, 124, 299

Cenarro, A. J., Gorgas, J., Cardiel, N., et al. 2001, Astrophys. Space Sci. Suppl., 277,319

Cenarro, A. J., Peletier, R. F., Sánchez-Blázquez, P., et al. 2007, MNRAS, 374, 664

Cesetti, M., Pizzella, A., Ivanov, V. D., et al. 2013, A\&A, 549, A129

Coelho, P., Bruzual, G., Charlot, S., et al. 2007, MNRAS, 382, 498

Colina, L., Bohlin, R. C., \& Castelli, F. 1996, AJ, 112, 307

Conroy, C., \& van Dokkum, P. 2012, ApJ, 747, 69

Conroy, C., Gunn, J. E., \& White, M. 2009, ApJ, 699, 486

Cox, C. 1999, Allen's Astrophysical Quantities (Springer)

Cushing, M. C., Rayner, J. T., \& Vacca, W. D. 2005, ApJ, 623, 1115

Falcón-Barroso, J., Sánchez-Blázquez, P., Vazdekis, A., et al. 2011, A\&A, 532 A95

Frogel, J. A., Persson, S. E., Matthews, K., \& Aaronson, M. 1978, ApJ, 220, 75

Gray, R. O., \& Corbally, J., C. 2009, Stellar Spectral Classification (Princeton University Press)

Houdashelt, M. L., Bell, R. A., Sweigart, A. V., \& Wing, R. F. 2000, AJ, 119, 1424

Koleva, M., Prugniel, P., De Rijcke, S., Zeilinger, W. W., \& Michielsen, D. 2009, Astron. Nachr., 330, 960

Kučinskas, A., Hauschildt, P. H., Ludwig, H.-G., et al. 2005, A\&A, 442, 281

Lançon, A., \& Wood, P. R. 2000, A\&AS, 146, 217

Lanz, T., \& Hubeny, I. 2003, ApJS, 146, 417

Lanz, T., \& Hubeny, I. 2007, ApJS, 169, 83

Lejeune, T., Cuisinier, F., \& Buser, R. 1997, A\&AS, 125, 229

Lejeune, T., Cuisinier, F., \& Buser, R. 1998, A\&AS, 130, 65

Maraston, C. 2005, MNRAS, 362, 799

Maraston, C., Strömbäck, G., Thomas, D., Wake, D. A., \& Nichol, R. C. 2009, MNRAS, 394, L107

Mármol-Queraltó, E., Cardiel, N., Cenarro, A. J., et al. 2008, A\&A, 489, 885

Meneses-Goytia, S., \& Peletier, R. F. 2012, in IAU Symp. 284, eds. R. J. Tuffs, \& C. C. Popescu, 32

Meneses-Goytia, S., Peletier, R. F., Trager, S. C., \& Vazdekis, A. 2015, A\&A, 582, A97 (Paper II)

Mouhcine, M., \& Lançon, A. 2002, A\&A, 393, 149

Pecaut, M. J., \& Mamajek, E. E. 2013, ApJS, 208, 9

Peletier, R. 1989, Ph.D. Thesis, Rijksuniversiteit Groningen

Peterson, R. C. 1977, in BAAS, 9, 604

Pickles, A. J. 1998, PASP, 110, 863

Rajpurohit, A. S., Reylé, C., Allard, F., et al. 2013, A\&A, 556, A15

Rayner, J. T., Cushing, M. C., \& Vacca, W. D. 2009, ApJS, 185, 289

Sánchez-Blázquez, P., Gorgas, J., Cardiel, N., \& González, J. J. 2006, A\&A, 457, 787

Schiavon, R. P., Caldwell, N., \& Rose, J. A. 2004, AJ, 127, 1513

Soubiran, C., Le Campion, J.-F., Cayrel de Strobel, G., \& Caillo, A. 2010, A\&A, 515, A111

Straizys, V., \& Kuriliene, G. 1981, Ap\&SS, 80, 353

Vazdekis, A., Cenarro, A. J., Gorgas, J., Cardiel, N., \& Peletier, R. F. 2003, MNRAS, 340, 1317

Vazdekis, A., Sánchez-Blázquez, P., Falcón-Barroso, J., et al. 2010, MNRAS, 404, 1639

Westera, P., Lejeune, T., Buser, R., Cuisinier, F., \& Bruzual, G. 2002, A\&A, 381, 524

Worthey, G., Faber, S. M., \& Gonzalez, J. J. 1992, ApJ, 398, 69

Worthey, G., Faber, S. M., Gonzalez, J. J., \& Burstein, D. 1994, ApJS, 94, 687

Yamada, Y., Arimoto, N., Vazdekis, A., \& Peletier, R. F. 2006, ApJ, 637, 200 